\title{
Stereotypes Disrupt Probabilistic Category Learning
}

\author{
Yrian Derreumaux ${ }^{1}$, Jacob Elder ${ }^{1}$, Gaurav Suri², Avi Ben-Zeev², Thelonious Quimby, \\ Brent L. Hughes ${ }^{1}$ \\ ${ }^{1}$ University of California, Riverside, ${ }^{2}$ San Francisco State University
}

\begin{abstract}
Author Note
This research benefited greatly from the input and feedback we received at several conferences and seminars, including posters that were presented at the Society for Personality and Social Psychology (2020), and the Social Affective Neuroscience Society (2019). We also thank Nick Camp (University of Michigan) and Jamil Bhanji (Rutgers University) for helpful discussion. Data and materials are publicly available at:

https://osf.io/fxkh7/?view_only=a24caf36566b418480560925a9e65f5c
\end{abstract}

Correspondence should be addressed to:

Brent Hughes

Department of Psychology

University of California, Riverside 900 University Ave, Riverside CA, USA

Email: bhughes@ucr.edu 
STEREOTYPES DISRUPT LEARNING

\begin{abstract}
Racial stereotypes exert pernicious effects on decision-making and behavior, yet little is known about how stereotypes disrupt people's ability to learn new associations. The current research interrogates a fundamental question about the boundary conditions of probabilistic learning by examining whether and how new learning is influenced by pre-existing associations. Across three experiments, participants learned the probabilistic outcomes of different card combinations based on feedback in either a social (e.g., forecasting crime) or nonsocial (e.g., forecasting weather) learning context. During learning, participants were presented with either taskirrelevant social (i.e., Black or White faces) or non-social (i.e., darker or lighter clouds) stimuli that were stereotypically congruent or incongruent with the social and nonsocial learning context. Participants exhibited learning disruptions in the social compared to nonsocial learning context, despite repeated instructions that the social and non-social stimuli were unrelated to the outcome (Studies 1-2). We also found no differences in learning disruptions when participants learned in the presence of negatively- (Black and criminal) or positively-valenced stereotypes (Black and athletic; Study 3). Finally, we tested whether learning decrements were due to "first order" stereotype application or inhibition at the trial-level, or due to "second order" cognitive load disruptions that accumulate across trials (Aggregated Analysis). We found no evidence of first order disruptions and instead found evidence for second-order disruptions: participants who were more internally motivated to respond without prejudice, and thus more likely to selfmonitor their responses, learned less accurately over time. We discuss the implications for the influence of stereotypes on learning and memory.

Keywords: stereotyping and prejudice, learning and memory, social cognition, internal motivation to respond without prejudice
\end{abstract}


STEREOTYPES DISRUPT LEARNING

Humans' ability to learn probabilistic associations through trial and error is key to survival. Consider the proverbial hot stove effect: A child might be told countless times to be careful playing around a stove without learning, but getting burned once can produce a lifelong association. Indeed, there exist numerous models of learning that account for how efficiently humans learn and update probabilities from subjective "hands-on" experiences (e.g., Cleeremans et al., 1991; Kumaran et al., 2016; Gobet et al., 2001; Lee et al., 2012). One important unanswered question is how the cognitive system handles different salient contextual cues during learning, such as pre-existing associations that are irrelevant to the learning context but may nonetheless impede new learning. For instance, police officers that deploy search and stop practices are tasked to learn objective indicators of suspicious behavior. However, research finds that officers often rely on pre-existing negative stereotypes to inform suspicions when conducting stops and searches (Minhas \& Walsh, 2021). Thus, negative pre-existing associations between Black men as criminal seem to interfere with police officers' ability to learn objective indicators of suspicion. Actively ignoring such disruptive pre-existing associations is an important challenge to the learning process (Niv et al., 2015). Here, we interrogate a fundamental question about the boundary conditions of probabilistic category learning: When are we able to ignore pre-existing associations that may interfere with learning and when can we not?

There are countless different kinds of pre-existing associations that people use daily that could disrupt learning new associations, including both social and nonsocial features of their environments. For instance, many people have strong associations between cloudy skies and rain and thus carry an umbrella or put on a raincoat when they see cloudy skies. As highlighted in the example above, people also have strong pre-existing associations about other people based on their demographics (e.g., race and gender), which may likewise influence decision-making and 
STEREOTYPES DISRUPT LEARNING

behavior (e.g., Brewer, 2001; Brewer \& Pierce, 2005; Hogg et al., 1995; Onorato \& Turner, 2004; Willer et al., 1989; Allidina \& Cunningham, 2021). Critically, extant research has not yet examined the influence of pre-existing associations on learning more broadly, or whether social and nonsocial association differentially influence new learning. One factor that may influence people's ability to ignore task-irrelevant associations is how "sticky" the association is. We use the term "stickiness" to describe associations that are resistant to change, difficult to inhibit, and may thus be more difficult to ignore (e.g., Arrow 1998; Blind et al., 2021).

There is good reason to believe that one class of social associations that people hold, namely racial stereotypes, may be particularly sticky and difficult to ignore, which may disrupt learning. Decades of research on stereotyping and prejudice show that racial associations powerfully influence decisions and behavior (e.g., Devine, 1989; Eberhardt et al., 2004; Welch, 2007; Simon, 1956; also see, Kirsch et al., 2004; Staddon \& Cerutti, 2003), such that the mere presence of an individual can instantaneously conjure up rich and affectively-laden mental representations based on their social category (Fiske \& Neuberg, 1990; Barnett et al., 2021; Schiller et al., 2009; Stephan \& Stephan, 1985). One such association that has garnered a great deal of attention in the United States is the stereotype that Black men are aggressive and dangerous (Eberhardt et al., 2004; Welch, 2007; Bodenhausen \& Wyer, 1985). For instance, research finds that people are more likely to misjudge a tool as a weapon when they are held by Black compared to White men (Correll et al., 2015), are quicker to shoot an armed target if they are Black compared to White (Correll et al., 2002; Payne, 2001), more readily associate aversive experiences (e.g., electric shocks) with Black compared to White individuals (Olsson et al., 2005), and assign harsher sentences to inmates with more Afrocentric features (Blair et al., 
STEREOTYPES DISRUPT LEARNING

2004). Together, this body of work suggests that this class of social associations may be particularly sticky and may thus impinge on learning.

At the same time, changes in the social milieu have reduced the social desirability and acceptability of expressing stereotypes, which has reduced explicit racial prejudice (Blinder et al., 2013). Consequently, people face personal pressures - due to internal egalitarian values and external social pressures to comply with social norms - to self-monitor and regulate the expression of stereotypes and prejudice (Butz et al., 2001; Plant \& Devine, 2003). This act of self-regulation to avoid appearing prejudiced has been shown to deplete subsequent cognitive functioning (Richeson et al., 2003; Richeson \& Shelton, 2003; Richeson \& Trawalter, 2005; Shelton et al., 2005; Rubien-Thomas et al., 2021). For instance, one study found that after an interaction with a Black confederate, White participants performed worse on a subsequent decision-making task (i.e., Stroop task), presumably due to depleted cognitive resources from self-regulation during the preceding interracial interaction (Richeson \& Shelton, 2003). Notably, this work focused on the effects of cognitive depletion from interracial interactions on subsequent task performance, rather than examining whether the pre-existing racial associations that people hold (e.g., stereotypes) disrupt new learning, in real time. As these two processes learning on the one hand and decision-making on the other - are categorically distinct, we do not yet know how such pre-existing associations disrupt the cognitive processes involved in learning.

The current work aims to fill this important gap by testing whether and how taskirrelevant social associations, such as racial stereotypes about Black men as threatening, disrupt probabilistic category learning, and directly compare whether the effects of social associations are stronger than task-irrelevant nonsocial associations, such as associations between clouds and rain. Consistent with a host of research demonstrating that stereotypes influence cognition and 
STEREOTYPES DISRUPT LEARNING

behavior, we predict that racial associations will disrupt the acquisition of new associations more than nonsocial associations. Regarding how social associations may prove disruptive, past research suggests at least two possible mechanisms.

First, stereotypes may exert 'first-order' effects that disrupt learning on a trial-by-trial basis due to stereotype application or inhibition. For instance, stereotypes that are irrelevant to a learning context may disrupt learning by increasing people's propensity to learn stereotype congruent over incongruent outcomes (i.e., stereotype application). That is, if people apply a stereotype (e.g., a person predicts that an individual has a characteristic ["is a criminal"] simply because they belong to a racial group [e.g., "is Black"]), then this will lead to suboptimal learning, as race is orthogonal to predictions about crime. Likewise, stereotypes may disrupt learning on a trial-by-trial basis by increasing people's propensity to inhibit stereotypically congruent responses. That is, if people inhibit stereotype-congruent responses (e.g., a person is reluctant to predict that an individual has a characteristic ["is a criminal"] simply because they belong to a racial group [e.g., "is Black"]), then this will also lead to suboptimal learning, as race is orthogonal to predictions about crime. An optimal learner should instead ignore task-irrelevant features of the learning environment.

In contrast to first-order stereotype application or inhibition, the presence of stereotypes may also disrupt learning via 'second-order' effects due to taxed cognitive functioning. Support for this hypothesis comes from past work showing that self-monitoring during interracial interactions can be cognitively taxing and thus diminish performance on subsequent cognitive control tasks (Richeson et al., 2003; Richeson \& Shelton, 2003). This hypothesis is also consistent with work demonstrating that extraneous cognitive load manipulations (e.g., divided attention) disrupt performance on cognitive tasks (Sweller, 2011). Thus, the act of self- 
STEREOTYPES DISRUPT LEARNING

monitoring one's behavior while also performing a learning task may disrupt learning. Moreover, if taxed cognitive functioning underpins the learning disruptions, then participants who are more internally motivated to respond without prejudice should perform worse than participants with lower internal motivations, as these individuals are more burdened by the additional cognitive effort of monitoring their responses (Johns et al., 2008). Individual differences are important predictors of a variety of real-world outcomes (Ozer \& Benet-Martínez, 2006), and may thus help to elucidate key mechanisms underlying probabilistic learning and their disruptions.

\section{The Current Studies: Experimental Paradigm and Predictions}

To examine whether and how pre-existing task-irrelevant associations disrupt probabilistic learning, we created a modified version of the weather prediction task (WPT; Knowlton et al., 1994). The WPT is a probabilistic learning task where participants learn to classify 14 different combinations of 4 cues (cards that depict geometric symbols) into categories (Sun or Rain). Each card or card combination maintains a different probability of predicting sun or rain, which participants learn over time based on feedback. Our motivation for using the WPT is two-fold. First, the WPT emulates multidimensional statistical features commonly found in real world learning, where people update learned associations over time based on discrete cues that are partially valid indicators of categorical outcomes (Krushke \& Johansen, 1999). Second, while learning during the WPT has traditionally been embedded within a nonsocial context (e.g., predicting rain or sun absent any social cues), the learning context can be adapted to include social cues (e.g., predicting crime in the presence of human faces). In doing so, we can directly test the extent to which contexts that elicit task-irrelevant social and nonsocial associations interfere with the learning process. Importantly, while there are many other kinds of social and 
STEREOTYPES DISRUPT LEARNING

nonsocial associations, here we focus on specific race-based social associations and weatherbased nonsocial associations.

Across experiments, task-irrelevant social stimuli consisting of Black and White images of male faces, and task-irrelevant nonsocial stimuli consisting of darker and lighter images of clouds, were introduced into a social learning context (predicting "steal" vs. "no steal") and nonsocial learning context (predicting "sun” vs. "rain”). By randomly presenting these images on a trial-by-trial basis, we introduced task-irrelevant stimuli that are either congruent or incongruent with one of the two possible predicted outcomes. For instance, the presence of a Black male face is stereotypically congruent with the prediction of "steal," just as the presence of darker rain clouds is congruent with the prediction of "rain." Conversely, the presence of a White male face is stereotypically incongruent with the prediction of "steal," just as the presence of lighter clouds is incongruent with the prediction of "rain." Thus, by introducing stimuli that are either congruent or incongruent with prior associations, we can examine the degree to which social and nonsocial associations interfere with learning. Critically, participants were repeatedly told that the social and nonsocial stimuli were distractors and should therefore be ignored. That is, the design created an expectation for participants to ignore these irrelevant distractors, and the empirical question is whether social associations are more difficult to ignore than nonsocial associations, and therefore disrupt learning.

Study 1 tests whether people learn differently in a social (i.e., forecasting crime) versus a nonsocial context (i.e., forecasting weather). Study 2 further tests whether learning disruptions in the social contexts is merely due to attention directed to distracting human faces or due to the unique combination of faces that were embedded within stereotype-eliciting contexts. Study 3 manipulates the valence of the learning context to examine whether stereotypes interfere with 
STEREOTYPES DISRUPT LEARNING

learning for both negatively-valenced and positively-valenced stereotypes. Next, we conduct an aggregated analysis that examines how social contexts disrupt learning. The aggregated analysis tests competing hypotheses regarding whether learning decrements are attributable to first order disruptions at the trial level due to stereotype application or inhibition, or to second order disruptions that accumulate over time due to taxed cognitive functioning. Study 2 was preregistered (see https://aspredicted.org/blind.php? $\mathrm{x}=\mathrm{n} 22299$ ).

\section{Study 1: Do Racial Versus Weather Associations Differentially Disrupt Learning?}

Study 1 was designed to test whether one class of social associations, namely stereotypes about race and crime, disrupt learning more than one class of nonsocial associations, namely associations between clouds and rain.

\section{Method}

Participants. Participants $(\mathrm{N}=114)$ were recruited from Amazon's Mechanical Turk (MTurk). They received $\$ 2.00$ payment for completing the roughly 20-minute study. Only MTurk workers with a history of providing good-quality responses were allowed to participate in the study (i.e., an acceptance rate of $>98 \%$ ). The sample size was based on prior research using the same task design (Gluck, 2002). ${ }^{1}$ Participants were excluded if they failed to achieve an average accuracy of $52 \%$, which indicated that they were randomly guessing ${ }^{2}$. After applying

\footnotetext{
${ }^{1}$ Gluck (2002) employed a repeated measures ANOVA to examine learning across four blocks of 50 trials, using a sample size of 30 participants. Given the large differences in our design (between vs. within subjects) and analyses (logistic mixed model vs. repeated measures ANOVA), we were unable to use the effect size observed in Gluck (2002). We instead relied on a judgement call (see e.g., Lakens, 2022) ultimately deciding to roughly double the sample size for our initial study.

${ }^{2}$ The exclusion criterion for accuracy was chosen during a group discussion with the idea that $52 \%$ was just enough above $50 \%$ to constitute some learning. This value refers to the mean accuracy across all trials, rather than a running average. Importantly, the results do not meaningfully change when we apply a 50\% accuracy threshold (see Table 9 in Supplemental).
} 
STEREOTYPES DISRUPT LEARNING

these exclusion criteria, $\mathrm{N}=100$ participants remained. The sample had a mean age of $37.30, S D$ $=11.07$ (56\% self-identified male, and 44\% self-identified female) and was $73 \%$ White, $10 \%$ Black, 10\% Asian, 4\% Latino, 3\% Other.

Task Design. The task was built using JavaScript and was hosted on biz.nf - a $3^{\text {rd }}$ party website (see OSF for script and pg. 2 of Supplemental Materials for a description of how the task was built). Across all studies, participants were asked to learn the probabilities of four cards (i.e., square, diamond, circle, and triangle), which were independently associated with each possible outcome (Weather: sun or rain; Crime: steal or no steal) at a fixed probability (Knowlton et al., 1994). In each trial, participants were presented with a particular combination of one, two or three of the four cards (never all four or none). There was a total of 14 possible card combinations used to generate 300 trials with different frequencies, in which the two outcomes occurred equally often (see Supplemental Table 1). The card or card patterns were pseudorandomized across trials to ensure they did not appear twice in succession.

The task-irrelevant race or sky images were displayed below each card or card combination. These images were also pseudo-randomized to ensure the same image did not appear more than twice in succession, but that each image had an equal probability of being displayed across all trials. The nonsocial stimuli consisted of four images of clouds that were gathered from free internet sources. These images were piloted and chosen based on the following criteria: (1) they could not be rendered in any way, (2) they could not contain rain or sun (i.e., only darker or lighter clouds), (3) they could not contain any land elements (i.e., only sky), and (4) they were roughly matched on how cloudy the sky images were. The social stimuli consisted of two White and two Black male faces selected from the Chicago Face Database (Ma 
STEREOTYPES DISRUPT LEARNING

et al., 2015), and were equated across various features (e.g., perceived attractiveness, aggressiveness, age; see Supplemental Table 2 \& 3).

Training. Participants received extensive instructions prior to the task (see pg. 3 in Supplemental for cover story). The cover story included an explanation as to why the stimuli were present during each trial, and that the social and nonsocial images presented below the card combinations were completely orthogonal to the task and non-diagnostic of the outcome, and therefore should not influence their choices. Importantly, participants were repeatedly told that the stimuli should be ignored and that they should instead focus on the cards. Participants were also informed that their choices early in the task would feel like random guesses, but that their performance through trial-by-trial feedback would increase over time. In addition, participants completed a total of six practice trials (see Figure 1). Thus, participants did have some exposure to card probabilities prior to the start of the task, which helps to explain why it appears that they are above chance at trial 1 . Participants were also required to correctly answer multiple choice comprehension check questions that repeated until a correct response was made (see pg. 3-4 in Supplemental for list of questions). These questions covered important features of the task to ensure validity and comprehension about the nature of the feedback (i.e., optimal choice vs. actual outcome [see below for more detail]), as well as the stimuli and learning context.

Procedure. After accepting the HIT on mTurk, participants were provided a link to the task. Once entered, they were given an informed consent detailing the risks and benefits of the study. Following the training phase, participants were instructed to predict whether the card or card combination would forecast "sun" or "rain" the next day (Weather condition) or "steal" or "no steal" the next day (Crime Face condition). Participants made their prediction by using their cursor to click on one of the two options (participants were ineligible to participate in the study if 
STEREOTYPES DISRUPT LEARNING

they were using a tablet or phone), which were displayed below the stimuli (the side of the screen where the buttons were presented were counterbalanced across participants; see Figure 1). In each trial, one card or card combination was presented on the top of the screen and participants had up to seven seconds to make their prediction. Failing to respond within this seven-second time window would automatically start the next trial, and participants were not provided any feedback. Three failed responses in a row would trigger a notification that asked participants if they were still paying attention. Clicking "yes" would resume the task where it left off. If participants did respond within seven second, they were provided feedback regarding whether they made the optimal choice or not (based on the cumulative probability of that card or card pattern) and what the actual outcome of that trial happened to be (based on the predetermined frequency of that pattern and the cumulative probability; see Supplemental Table 1). An optimal choice represents any prediction where the card or card patterns are greater than $50 \%$ of the predicted outcome. If participants made the optimal choice, they were shown a green check mark (correct) on the right side of the stimuli, whereas if participants made the suboptimal choice, they were shown a red $\mathrm{x}$ (incorrect).

To monitor overall performance on the task, each correct response corresponded with a one unit increase in a green bar on the right side of the screen, whereas each incorrect response corresponded with a one unit increase in a red bar. In addition to seeing feedback about whether or not the optimal choice was made, participants were also provided feedback about the actual outcome that trial. This feedback was presented in text (Weather: sun vs. rain; Crime: steal vs. no steal) on the left of the stimuli. Finally, participants were also provided with a progress bar on the bottom of the screen. 
STEREOTYPES DISRUPT LEARNING

Analysis plan. To test whether racial associations disrupt learning more than weather associations, we fit a logistic mixed model regressing accuracy $(0=$ incorrect; $1=$ correct $)$ onto a dummy coded factor representing the fixed effect of condition $(0=$ Crime Face $[$ reference group]; 1 = Weather Cloud), and a variable representing time (i.e., trial). The time variable was scaled and estimated with random slopes for trials within subjects. To examine whether learning trajectories changed over time as a function of the learning environment, we included a time by condition interaction. In addition, to ensure generalizability of our stimuli, we modeled stimuli and participants as random factors. The full model included four fixed effects (intercept, main effect of trial, main effect of condition, and trial by condition interaction) and three random effects (stimuli, participant, trial). This mixed model was estimated using the lme4 package in $\mathrm{R}$ (Bates et al., 2014), with $p$-values generated with Satterthwaite approximation using the lmerTest package (Kuznetsova et al., 2017). 


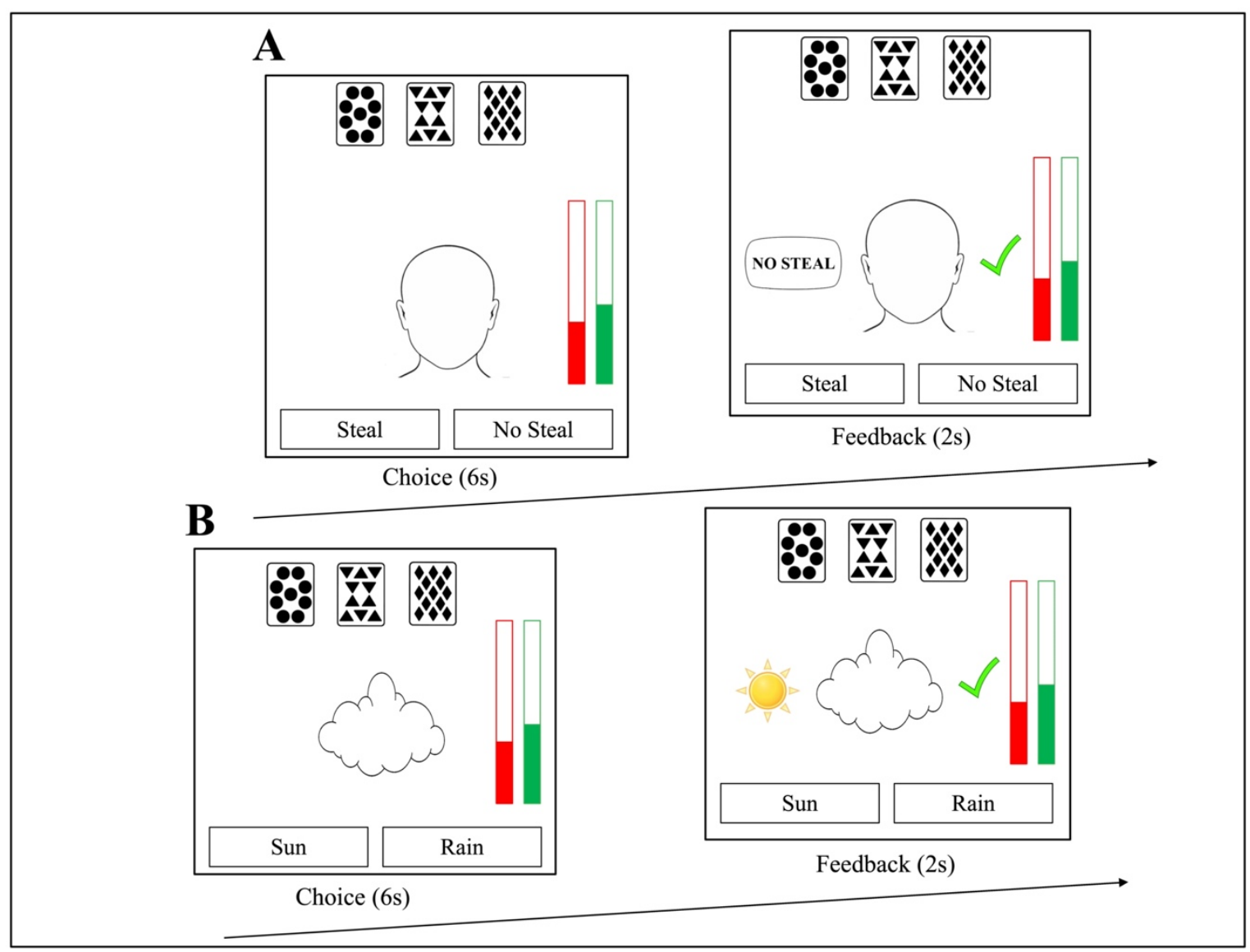

Figure 1. Schematic of the learning task in the Crime Face (A) and Weather Cloud (B) conditions. Participants learned the probabilities of the card patterns by selecting their choice and receiving feedback as to if they were correct or incorrect (right side of stimuli) as well as what the actual outcome was on that trial (left side of stimuli). Note* that the stimuli in this diagram were the same stimuli shown to participants during the practice trials.

\section{Results}

This model revealed two significant main effects. First, we observed a main effect of Trial $(b=.45,95 \% C I=[.35, .55], S E=.05, z=8.63, O R=1.5, p<.0001)$, demonstrating that participants became more accurate as the task progressed. Second, we observed a significant main effect of condition $(b=-.33,95 \% C I=[.035, .67], S E=.13, z=2.34, O R=1.42, p=$ .026), demonstrating that participants in the Crime Face conditioned performed worse relative to participants in the Weather condition (see Figure 2). We did not observe a significant Trial by 
STEREOTYPES DISRUPT LEARNING

Condition interaction $(b=.09, S E=.008, z=8.63, O R=1.2, p=.23)$.

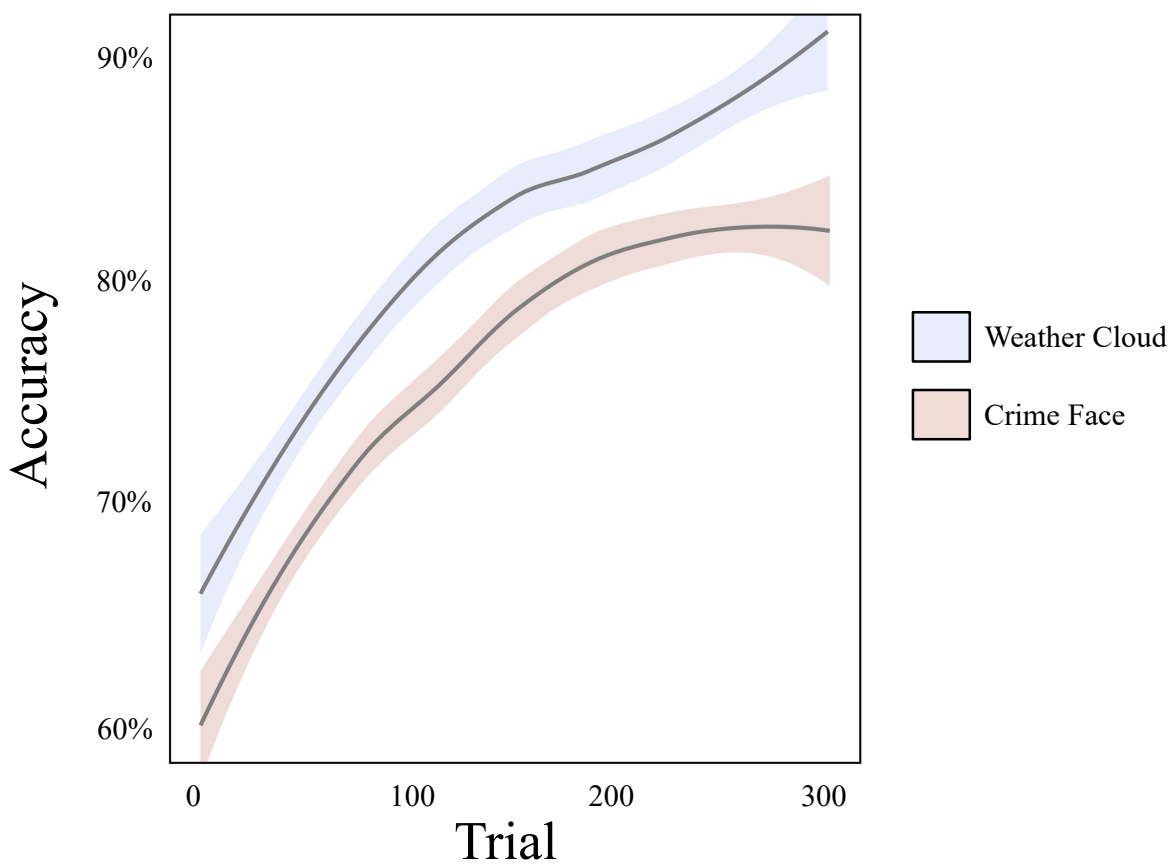

Figure 2. learning rates (loess) as a function of condition. The blue line denotes participants in the Weather Cloud condition, whereas the brown line denotes participants in the Crime Face condition. Note * that the error bars denote standard error of the mean.

\section{Discussion}

Study 1 provides preliminary evidence that social associations about race and crime disrupt learning more than nonsocial associations about weather. This is an intriguing finding considering that people maintain associations between clouds and weather outcomes just as they maintain racial associations. These findings also raise the question of whether learning decrements are attributable to the mere presence of faces, or whether it was the combination of faces within a context that elicits stereotypical associations that influences learning. People are highly attuned to human faces from the first days of life (Frank et al., 2009; Barnett et al., 2021), and racial outgroup faces in particular have been shown to inadvertently garner greater attention 
STEREOTYPES DISRUPT LEARNING

and eye-gaze relative to ingroup faces (Trawalter et al., 2008). Across time, divided attention may contribute to suboptimal learning.

The interpretation of Study 1 results was also limited by a potential confound in the weather stimuli, due to the images of clouds being more strongly associated with sun than rain (see Supplemental Table 4). That is, the weather stimuli were not balanced in terms of their associations with outcomes of sun and rain. In contrast, the race stimuli were balanced in that half the images depicted a Black male face and the other half a White male face. In addition, it is unclear whether learning decrements are attributable to the forecasting context, whereby one kind of prediction (e.g., crime) is simply stranger than the another (e.g., weather). Thus, we designed Study 2 to address these confounds and alternative explanations.

\section{Study 2: Separating the Influence of Human Faces from Stereotypes on Learning}

Prior research shows that people are highly attuned to human faces, and that their presence inadvertently captures attention (Theeuwes \& Van der Stigchel, 2006). Thus, the mere presence of faces may inadvertently garner attention and thereby disrupt optimal learning. This effect is stronger when people are presented with faces of racial outgroups compared to racial ingroups (Trawalter et al., 2008). Study 2 was designed to replicate and extend Study 1 by dissociating learning decrements that may be due to the presence of human faces from stereotypical associations, in addition to addressing potential confounds in the cloud stimuli used in Study 1. To that end, Study 2 included two additional conditions that crossed the stimuli and learning context. If learning decrements are due to the presence of faces alone, then we would expect no difference in learning for participants presented with faces in the context of crime compared to participants presented with faces in the context of weather. 
STEREOTYPES DISRUPT LEARNING

\section{Method}

Participants. Participants $(\mathrm{N}=393)$ were recruited from the University of California SONA pool, and received course credit for completing the study. Participants were once again excluded based on a priori exclusion criterion of 52\% accuracy (see Supplemental Tables 6-8 for breakdown of excluded participants by condition). After applying these exclusion criteria, $\mathrm{N}=$ 373 participants remained. The sample had a mean age of $20.01, S D=3.32(63 \%$ self-identified female, and 37\% self-identified male) and was $43 \%$ Asian, $28 \%$ Latino, $11 \%$ White, $8 \%$ Other, and $6 \%$ Black.

We conducted a post hoc sensitivity analysis using the Simr package (Green \& Macleod, 2016) in $\mathrm{R}$ which revealed an observed power of $.93,95 \% C I[.86, .97]$ to detect the main effect of Condition in Study 2, demonstrating sufficient power.

Task Design. The configuration of the paradigm, including the placement of the card or card patterns, the race and weather stimuli, feedback, and the progress bar were all identical to Study 1. The script was once again hosted on biz.nf. One notable change is that Study 2 reduced the total number of trials from 300 to 200 to reduce participant fatigue given the length of the task.

One limitation of Study 1 was that the weather stimuli were, on average, more strongly associated with sun. Study 2 included a new and larger set of weather stimuli that were balanced in terms of their associations of sun and rain. Specifically, 30 images of clouds were piloted in a separate sample $(\mathrm{N}=22)$, and eight images were selected for Study 2, four of which were more strongly associated with rain and four that were more strongly associated with sun (see Supplemental Table $4 \& 5$ ). The race stimuli were once again selected from the Chicago Face 
STEREOTYPES DISRUPT LEARNING

Database, but included the addition of four new faces images from the database to increase our sample of stimuli (see Supplemental Table 3).

Training. Participants once again received extensive instructions prior to the task to ensure validity and received the same comprehension checks as participants in Study 1.

Procedure. The procedure was similar to Study 1 but included two additional conditions that were designed to control for context and stimulus-driven effects. Specifically, participants were also randomly assigned into a condition where they were asked to (1) predict "sun" or "rain," while seeing images of Black and White faces (Weather Face), or (2) predict "steal” or “no steal," while seeing images of clouds (Crime Clouds). In all, participants were subject to a 2 (context: Crime vs Weather) by 2 (stimuli: Faces vs. Clouds) design. Thus, if learning decrements are due to the presence of faces alone, then we would expect no difference in learning between the Crime Face and the Weather Face condition.

Analysis plan. To examine differences in learning across the four conditions, we estimated a logistic mixed model regressing accuracy onto a dummy coded factor for condition (Weather Face condition $=0$ [reference group]), as well as a continuous variable for time. The time variable was scaled and estimated as a random slope for trial within subjects. To further test whether learning decrements varied as a function of time, and if the effect of time depended on the learning environment (i.e., condition), the full model included a time by condition interaction term. Both stimuli and participants were once again estimated as random factors to increase generalizability.

To test the unique influence of faces from faces embedded within contexts that elicit stereotypical associations, we further conducted pairwise simple contrasts between the Crime condition and all other conditions. 
STEREOTYPES DISRUPT LEARNING

Finally, to test whether self-identified participant race moderated learning, we also report mixed models that test the interaction of race by condition. Given that our sample in Study 2 contained a relatively small proportion of self-identified "White", "Black" and "Other" participants, we report one model where each race category is coded as a separate factor (effects coded with "Other" coded as -1), as well as a second model where "White", "Black" and "Other" are collapsed into a single "Other" factor. ${ }^{3}$

\section{Results}

Replicating Study 1 and in line with our pre-registered hypothesis, we observed one significant main effect of condition (see Figure 3), demonstrating that participants in the Crime Face condition $(M=.77, S D=.42)$ were significantly less accurate relative to participants in the Weather Cloud condition $(M=.82, S D=.38 ; b=-.36,95 \% C I=[-.57,-.15], S E=.11, z=-3.36$, $O R=69, p=.0007)$. Notably, we did not observe a significant difference between participants in the Weather Face and Weather Cloud condition $(b=-.10, S E=.107, z=-.94, p=.34)$, or between participants in the Crime Cloud and Weather Cloud condition $(b=-.15, S E=.105, z=$ $1.46, p=.14)$

Simple contrasts revealed that participants who made prediction about crime in the presence of faces learned significantly worse than participants who made predictions about crime in the presence of clouds $(b=-.25, O R=.77, S E=.108, z=-2.40, p=.01)$, and learned marginally worse than participants who made prediction about weather in the presence of faces $(b=-.20, O R=.81, S E=.10, z=1.93, p=.053)$. To quantify the practical implication of the latter effect size, we calculated the percent accuracy change between participants in the Crime

\footnotetext{
${ }^{3}$ Individual differences (e.g., motivation to respond without prejudice, social dominance orientation) were also collected in studies 2 and 3, and these data will be reported in an aggregated analysis later in the manuscript.
} 
STEREOTYPES DISRUPT LEARNING

Face and Weather Face condition. To do so, we set the Crime Face condition as the base rate for learning performance $(4286$ [incorrect $] /(14028$ [correct $]+4286$ [incorrect $]=0.23)$, and then used the odds ratio representing the difference in learning between the Crime Face and Weather Face (.81) to calculate the relative difference in error rates between these two conditions (see pg. 13 in Supplemental for formula). Doing so revealed an incorrect rate of $14.69 \%$ in the Weather Face compared to $23.40 \%$ in the Crime Face condition. In practical terms, the error rate increases more than $50 \%$ when participants learn in an environment where faces are stereotypically associated with predicted outcomes relative to when they are not. These results suggest that while the presence of faces does impede new learning, the combination of faces in a context that elicits racial associations with crime leads to larger learning disruptions.

Finally, we tested whether participant race moderated learning and found no significant difference in accuracy across conditions as a function of self-identified race (all $p$-values $>.180$; see Table 10 in Supplemental for full model output). These effects did not meaningfully change when race was collapsed into a three-level factor compared to a five-level factor (all $p$-values $>$ .185; see Table 11 in Supplemental for full model output). 


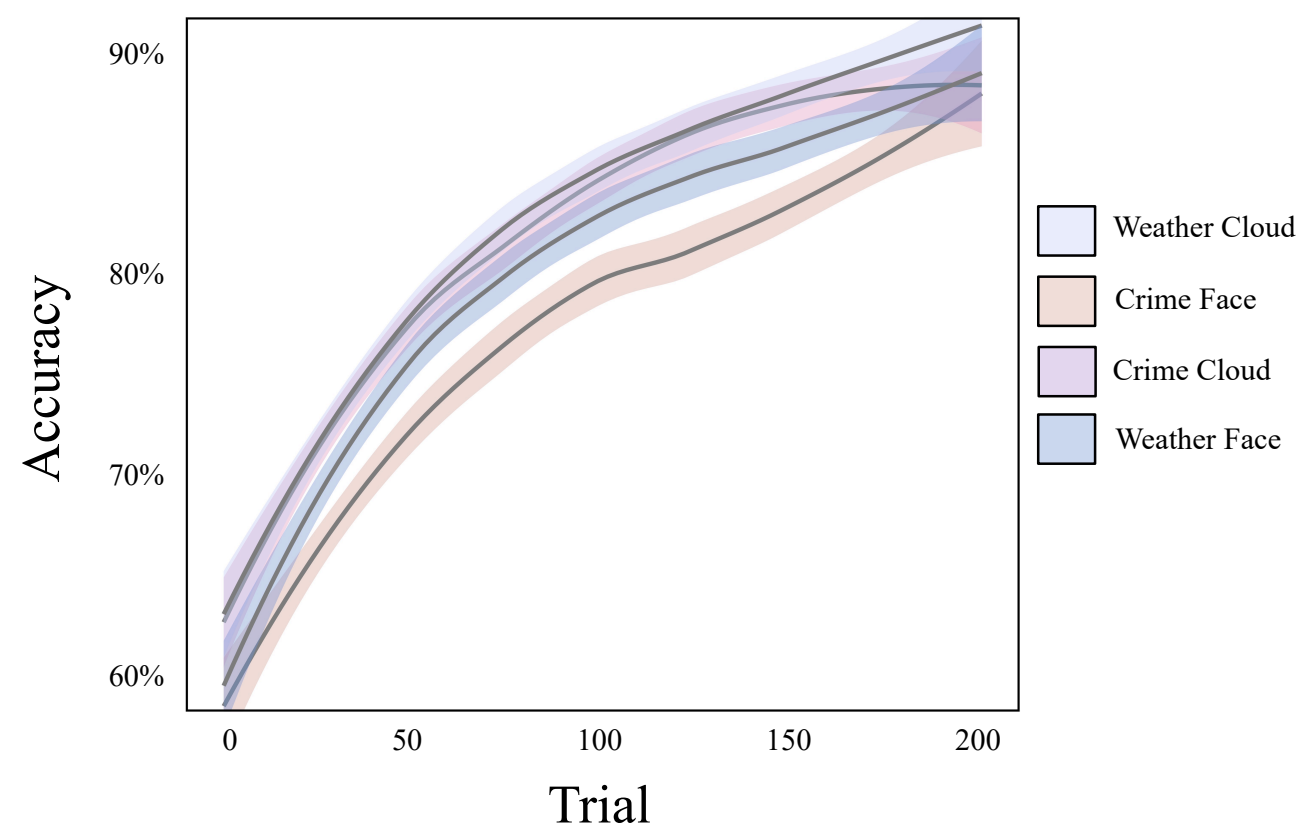

Figure 3. Learning rates (loess) as a function of condition. Note* that the error bars denote standard error of the mean.

\section{Discussion}

Study 2 replicated results from Study 1 and found that learning in the presence of racial associations disrupted learning more than learning in the presence of weather associations. By crossing the stimuli and the learning context, Study 2 also dissociated the unique effect of faces on learning. Doing so revealed that error rates in the Crime Face increased by over $50 \%$ compared to error rates in the Weather Face condition, suggesting that the presence of racial stereotypes in the learning context may additionally disrupt learning over the mere presence of faces. These results highlight the impact of stereotypes on probabilistic category learning and updating, and suggest that even when people are explicitly told that the racial stimuli have no association with the outcome, they still negatively impact learning.

One notable difference between Studies 1 and 2 pertained to the sample demographics. Study 1 was conducted with a majority White sample (73\% White), whilst Study 2 was 
STEREOTYPES DISRUPT LEARNING

conducted with a majority minority sample (11\% White). Importantly, participant race did not moderate learning in Study 2, which is consistent with past work showing that stereotypes are culturally shared and influence cognition across myriad racial and ethnic groups (Axt et al., 2014).

\section{Study 3: Do Learning Decrements Extend to Positively-Valenced Stereotypes?}

Study 2 provided converging evidence that social contexts that elicit negative racial associations disrupt learning more than nonsocial contexts that elicit weather associations. Notably, learning in the Crime Face condition involves one of the most salient and harmful stereotypes in the U.S. of Black men as criminal (Welch, 2007). Thus, learning may be uniquely disrupted in this and other contexts related to intergroup threat (Chang et al., 2016), relative to contexts that elicit stereotypes that people perceive to be more innocuous. This begs the question of whether similar learning decrements would arise in contexts that elicit positively-valenced stereotypical associations, for instance stereotypes about Black men as athletic.

It is important to note that we refer to positively-valenced stereotypes as those that are subjectively perceived to be favorable characteristics of a group, rather than those that are experienced as positive by members of the stereotyped group, or that lead to positive outcomes for the stereotyped group. Indeed, research finds that targets of positive stereotypes experience similar emotional responses-dislike, resentment, and negativity-as targets of negative stereotypes (Siy \& Cheryan, 2016; Czopp, 2008). Moreover, people who tend to endorse negative stereotypes about Black Americans also tend to exhibit stronger stereotypes endorsing Black as athletic (Kurdi et al, 2019; see also Kay et al., 2013), suggesting that these two classes of stereotypes may similarly influence intrapersonal and interpersonal psychological processes. Due to their "complementary" nature, positive stereotypes are often treated as innocuous or even 
STEREOTYPES DISRUPT LEARNING

flattering (Bergsieker et al., 2012), but they have been shown to be as harmful as negative stereotypes, especially due to their pervasiveness and general acceptance in the social milieu (Czopp et al., 2015; Devine \& Elliot, 1995). As such, people are more likely to endorse positive relative to negative stereotypes, and people who publicly endorse positive stereotypes are seen as less prejudiced than people who publicly endorse negative stereotypes (Mae \& Carlston, 2005).

Given that people may be less concerned about appearing prejudiced in the context of positive stereotypes, one possibility is that they may exert less of an influence on learning. On the other hand, any stereotypical association may continue to disrupt learning because of its salience, and because people may want to avoid endorsing any kind of race-based stereotype. Study 3 thus sought to extend Study 2 and test whether learning decrements extend to contexts that elicit positive stereotypes about Black men as athletic.

\section{Method}

Participants. Participants $(\mathrm{N}=220)$ were recruited from the University of California SONA pool, and received course credit for completing the study. Participants were once again excluded based on a priori exclusion criterion of $52 \%$ accuracy. After applying these exclusion criteria, $\mathrm{N}=206$ participants remained. The sample had a mean age of $19.43, S D=1.86,(62 \%$ self-identified female, and 38\% self-identified male) and was $45 \%$ Asian, $32 \%$ Latino, $10 \%$ White, $7 \%$ Other, $3 \%$ Black.

Task Design. The structure of the paradigm, including the placement of the card or card patterns, stimuli, feedback and progress bar were all identical to Study 2, and the script was once again hosted on biz.nf. The social stimuli were also identical to Study 2 in both conditions and participants received extensive instructions prior to the task and received the same comprehension checks. 
STEREOTYPES DISRUPT LEARNING

Procedure. The procedure was similar to Study 2, however this time participants were either randomly assigned into a condition that elicited a positive stereotypical association (i.e., Black and athletic) or a context that elicited negative stereotypical associations (i.e., Black and criminal). Specifically, participants were either randomly assigned to a condition where they were asked to predict a "steal" or "no steal" outcome from card combinations, or a condition where they were asked to predict a "touchdown" or "no touchdown" outcome from card combinations. In the positive stereotype condition, optimal feedback was once again presented via a green check mark for correct prediction and a red $\mathrm{x}$ for incorrect predictions. The actual outcome was presented in text ("touchdown" vs. "no touchdown"). The negative stereotype condition was identical to Study 2. After the learning task, participants responded to the same individual differences measures as participants in Study 2. The stimuli (Black and White male faces) were identical to that in Study 2.

Analysis Plan. To test whether positive or negatively-valanced stereotypes differentially disrupt learning, we fit a logistic mixed model similar to that in Study 1. Specifically, we regressed accuracy $(0=$ incorrect; $1=$ correct $)$ onto a dummy coded factor representing the fixed effect of condition $(0=$ Crime Face [reference group]; $1=$ Athletic $)$, and a variable representing time (i.e., trial). The time variable was once again scaled and estimated with random slopes for trials within subjects. The full model included four fixed effects (intercept, main effect of trial, condition, and a trial by condition interaction term) and three random factors (stimuli, participants, and trial within subjects). To test whether participant race moderated learning, we also report a model that includes an interaction between self-identified race and condition. Each race category was coded as a separate factor (effects coded with "Other" coded as -1). 


\section{STEREOTYPES DISRUPT LEARNING}

Finally, to test the likelihood that the observed null effect of condition was true, we estimated a multilevel Bayesian logistic model using the brms package in R (Bürkner, 2017) with uninformative priors. One benefit of this approach is that it provides a more appropriate test for the probability of obtaining a null effect based on the proportion of the posterior distribution of the parameter estimate that falls within a range that would be considered negligible (i.e., the region of practical equivalence; see Krueshke, 2010, 2018). This model contained the exact same fixed and random effects as before.

\section{Results}

In the first model, we examined differences in learning across conditions and found no significant difference in learning between participants in the Crime Face and Athletic condition $(b=.048, S E=.11, z=.44, p=.66$; see Figure 4). Likewise, we found no evidence that accuracy differed across condition as a function of participant race (all $p$-values $>.14$; see Supplemental Table 12 for full model output).

In the second model, we tested the likelihood that the null was true, and demonstrated that participants in the Athletic condition had a $54.57 \%$ probability of being less accurate than participants in the Crime Face condition (Median of the parameter estimate $=-.01,89 \% C I$ $[-.23,19])$. More importantly, $100 \%$ of the posterior distribution fell inside the region of practical equivalence (see Supplemental Figure 2 for credible intervals), demonstrating a high likelihood for the null effect of condition. This null effect is intriguing because it suggests that although people are often less concerned about expressing positively-valenced stereotypes, their presence may still impact learning similar to negatively-valenced stereotypes. 


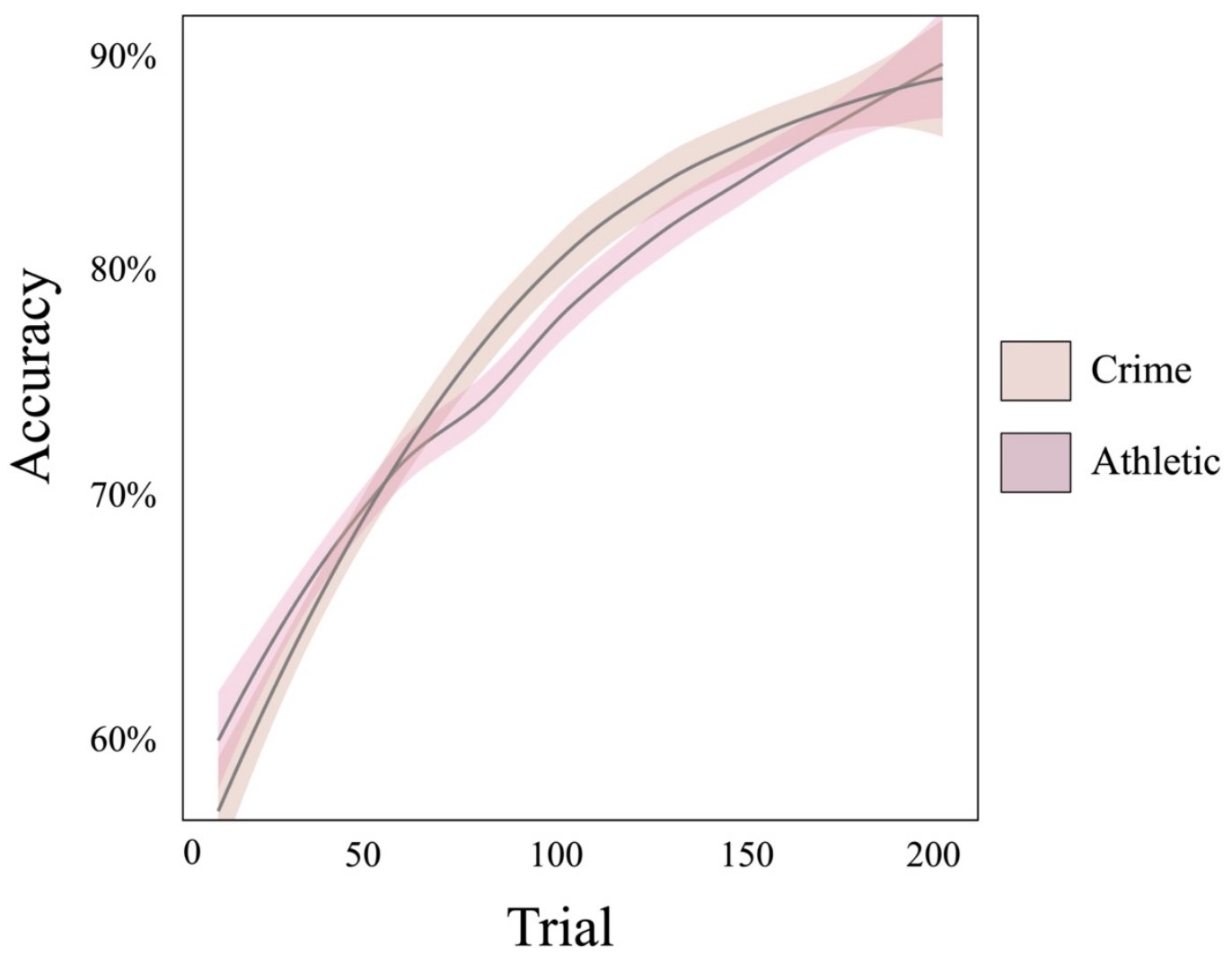

Figure 4. Learning rates (loess) as a function of condition. Note* that error bars denote standard error of the mean.

\section{Discussion}

Study 3 examined whether learning decrements extend to social contexts that elicit positively-valenced stereotypes about Black men as athletic. Interestingly, we found no difference in learning rates between the positive and negative stereotype condition.

One possible explanation for why we do not find difference in learning between the Athletic and Crime condition may be that the saliency of both stereotypes leads people to inadvertently incorporate them into their predictions, thereby disrupting learning on a trial-bytrial basis by weighting predictions towards stereotypically congruent outcomes. Alternatively, 
STEREOTYPES DISRUPT LEARNING

the mere presence of a stereotype, and the added cognitive effort of monitoring one's responses to avoid appearing prejudiced, may increase cognitive load and disrupt learning on a more cumulative basis.

\section{Aggregated Analysis: Testing Competing Mechanisms Underlying Learning Decrements}

We find converging evidence that task-irrelevant racial associations disrupt probabilistic learning above and beyond weather associations (Studies 1-2). Notably, we found no difference in learning between the positively (Black and athletic) and negatively (Black and criminal) valenced conditions (Study 3). Next, we interrogate two plausible underlying mechanisms based on past research.

First, stereotypes may disrupt learning via first-order stereotype application or inhibition, which occur at the trial level. Consistent with research demonstrating that people often automatically apply stereotypes during decision-making tasks (Eberhardt et al., 2004), stereotypes may similarly disrupt learning by increasing people's propensity to respond in a stereotype-congruent manner (i.e., stereotype application). For instance, participants may be more likely to predict "steal" when a Black face is present compared to a when a White face is present. Conversely, stereotypes may disrupt learning if people actively try to suppress their influence over predictions (i.e., stereotype inhibition). For instance, in an attempt to appear unprejudiced, participants may try to control their responses and actively suppress "steal" predictions when a Black face is present. Given that the faces are orthogonal to the cumulative probability of the card patterns, any choice that is influenced by faces should lead to suboptimal predictions on a trial-by-trial basis.

Second, stereotypes may disrupt learning via second-order effects that accumulate over time due to the cognitive effort elicited by the presence of a salient stereotype. This hypothesis is 
STEREOTYPES DISRUPT LEARNING

motivated by research suggesting that regulating the expression of prejudice during interracial interactions (Richeson et al., 2003) and processing task-irrelevant race information is cognitively taxing (Rubien-Thomas et al., 2021). Moreover, given that the stereotypes presented in the current experiments relate to racial inequities, we speculate that the influence of stereotypes on learning may be greatest for individuals who are more motivated by egalitarian values (e.g., racial equity). Racial stereotypes may be particularly salient for individuals who are more conscious of their negative effects (Johns et al., 2008), and who actively work to reduce their own racial biases (Devine et al., 2002). If stereotypes disrupt probabilistic learning by increasing cognitive effort needed to monitor responses, then individuals higher (versus lower) in internal motivations to respond without prejudice should perform worse on the current task. Such individuals are more burdened by the cognitive effort required to monitor their responses. ${ }^{4}$ Motivations to respond without prejudice are composed of separable and largely independent underlying components: an internal and external motivation (Devine et al., 2002; Plant \& Devine, 1998). Internal motivations (IMS) reflect the implications of appearing prejudiced to one's sense of self (e.g., "I attempt to act in nonprejudiced ways toward Black people because it is personally important to me"), and thus assess personal motivations that are not incumbered by external environmental factors. External motivations (EMS) focus on external social pressures that arise from normative pressures to not appear prejudiced (e.g., "I attempt to appear not prejudiced toward Black people in order to avoid disapproval from others").

\footnotetext{
${ }^{4}$ We pre-registered a series of reinforcement learning (RL) models to test competing mechanisms underlying learning. These RL models were consistent with our behavioral findings, such that we found no evidence for "firstorder" effects on learning, and instead found evidence for "second-order" effects in the RL models. We felt that these analyses do not provide greater nuance to the behavioral evidence and therefore they are reported in Supplemental (pgs. 19-25).
} 
STEREOTYPES DISRUPT LEARNING

Importantly, both IMS and EMS may moderate learning via different mechanisms, yet research has yet to examine if and how IMS and EMS influence learning. To ensure sufficient power and generalizability, we aggregated data across conditions and conducted an aggregated analysis (Goh et al., 2016; Eisenhauer, 2021).

\section{Method}

Participants. We selected all participants in the Crime Face $(\mathrm{N}=204)$ and Athletic conditions $(\mathrm{N}=93)$ across Study 2 and $3 .{ }^{5}$ Study 1 was not included in this analysis because we did not collect individual differences for participants in that sample. This sub-sample had a mean age of $19.43, S D=1.86,(62 \%$ self-identified female, and $38 \%$ self-identified male) and was $45 \%$ Asian, 32\% Latino, 10\% White, 7\% Other, 3\% Black.

\section{Self-report measures ${ }^{6}$}

Internal Motivation to Respond Without Prejudice. A 5-item questionnaire that asses a person's internal motivation to respond without prejudice (Plant \& Devine, 1998). The scale demonstrated strong reliability in the current sample (Omega $=.85)$.

External Motivation to Respond Without Prejudice. A 5-item questionnaire that asses a person's external motivation to respond without prejudice (Plant \& Devine, 1998). The scale demonstrated moderate to strong reliability in the current sample (Omega $=.79)$.

Social Dominance Orientation. A 16-item questionnaire that asses a person's preference for inequality among social groups (Pratto et al., 1994). The scale demonstrated strong reliability in the current sample $($ Omega $=.81)$.

\footnotetext{
${ }^{5}$ Note that the Athletic condition only applies to Study 3 .

${ }^{6}$ Several other individual difference measures were collected for exploratory purposes. These include the extraversion facet from the Big Five Inventory (Soto \& John, 2017), the honesty-humility facet from the HEXACO model of personality (Ashton \& Lee, 2007; Ashton et al., 2014), intergroup anxiety (Stephan \& Stephan, 1985), and the General Intergroup Contact and Quantity and Quality scale (Islam \& Hewstone, 1993). See pg. $7 \& 8$ as well as Figure 1 in Supplemental for more details.
} 
STEREOTYPES DISRUPT LEARNING

\section{Analysis plan.}

First order effects: stereotype application versus stereotype inhibition. To test whether learning decrements are due to stereotype application or inhibition, we fit a series of mixed models interrogating the influence of various trial level features on responses separately for participants in the Crime Face $(\mathrm{N}=204)$ and Touchdown $(\mathrm{N}=93)$ conditions. This included regressing accuracy onto a fixed effect of stimuli representing the race of the face present on a given trial (dummy coded with Black faces as reference group), which would help to determine whether accuracy was systematically influenced by the race of the face present (see Model 1 in Supplemental Table $13 \& 14)$. We also regressed a congruency variable onto reaction time and accuracy (see Model $2 \& 3$ in Supplemental Table $13 \& 14$ ). The congruency variable was set to 1 when a participant's prediction was stereotypically congruent (e.g., Black and steal), and 0 (reference group) when a participant's prediction was stereotypically incongruent (e.g., White and steal). This model would determine whether participant's predictions were systematically influenced by stereotype congruent compared to stereotype incongruent trials, for instance via less accurate or more rapid responses on stereotypically congruent trials. We also regressed the congruency variable onto the fixed effect of the predictive weight of the card patterns. Predictive weight was modeled both as an absolute deviation from .5 , providing a continuous measure of ambiguity (see Model 4 in Supplemental Table $13 \& 14$ ), as well as a categorical (effects coded) variable, which would indicate differences based on high vs. low predictive trials (see Model 5 in Supplemental Table $13 \& 14$ ). This model would determine whether participants were more likely to rely on the stereotype when the cards were less determinant of the outcome. Finally, we regressed the congruency variable onto individual differences that may moderate stereotype application or inhibition (e.g., Social Dominance Orientation, Pratto et al., 1994; IMS and EMS) 
STEREOTYPES DISRUPT LEARNING

(see Model $6 \& 7$ in Supplemental Table 13 \& 14). All models included a random factor for stimuli, as well as a time (trial) variable that was scaled and estimated as a random slope for trial within subjects. This time variable was first estimated as a covariate, and then subsequently as a moderator. Each model was tested both for participants in the Crime Face (see Supplemental Table 13) and Athletic conditions (see Supplemental Table 14). All models examining behavior in the Crime condition included a dummy coded covariate to control for study.

Second order effects: cumulative learning decrements over time. If learning is not disrupted on a trial-by-trial basis, but instead accumulates over the course of the task due to tax cognitive functioning, then participants who are higher on internal motivations to respond without prejudice should perform worse relative to those lower. Moreover, this added cognitive effort may result in longer response times for those who score highest on motivation to respond without prejudice ${ }^{7}$. To test these questions, we first estimated separate mixed models regressing accuracy onto a measure of internal (IMS) and external (EMS) motivation to respond without prejudice (see Model $1 \& 2$ in Supplemental Table $15 \& 16$ ). We also tested a model that included both IMS and EMS to examine the unique effects of IMS above and beyond EMS, as well as the interactive effects of the two (see Model 3 in Supplemental Table $15 \& 16$ ). Further, to test whether internal motivations to respond without prejudice was associated with longer response times, we estimate a model that regresses $\log$ (reaction time) onto IMS, while controlling for EMS (see Model 4 in Supplemental Table $15 \& 16)^{8}$. Finally, we also test

\footnotetext{
${ }^{7}$ We attempted to collect a measure of stereotype misperception (Krieglmeyer \& Sherman, 2012) but due to a coding error this data was sadly unusable and therefore not analyzed. As such, our pre-registered hypothesis for IMS (H2) could not be directly tested. Instead, we test the effects of IMS on learning over time, which is consistent with the pre-registered hypothesis.

${ }^{8}$ We also estimated a model for participants in Study 2 to examine whether the effect of IMS was specific to the Crime Face condition, or whether it extended to any context with faces (e.g., Weather Face). This model demonstrated that the effect of IMS on learning was unique to the Crime Face condition (Supplemental Figure 4).
} 
STEREOTYPES DISRUPT LEARNING

whether response times differed as a function of the race of the stimuli present on each trial, we regressed $\log ($ reaction time) onto a dummy coded factor for stimuli (Black $=0$ [reference group]; White $=1$ : see Model 5 in Supplemental Table $15 \& 16$ ). All continuous variables were scaled, and each model tested the interaction of the fixed effects with time. Once again, all models included random factors for stimuli, participants, and trial within participants. Each model was tested both in the Crime Face (see Supplemental Table 15) and Athletic condition (see Supplemental Table 16).

\section{Results}

First order effects in the Crime Face condition. Across all models, we found no evidence that learning was disrupted on a trial-by-trial basis for participants in the Crime Face condition. For instance, there was no difference in accuracy as a function of the race of the stimuli $(b=-.002, S E=.03, z=-.071, p=.94)$, no difference in accuracy or reaction time for stereotype-congruent versus incongruent trials $(b=.037, S E=.02, z=1.13, p=.16$, and $b=$ $.003, S E=.004, t=.064, p=.94$, respectively), nor were there any differences in the predictive weights of the card patterns for stereotype-congruent versus incongruent trials, regardless of whether card pattern weight was modeled as categorical (all $p$-values $>.11$ ) or as an absolute difference from $.5(b=.013, S E=.01, z=.1 .27, p=.20)$. Moreover, individual differences associated with increased stereotype application (i.e., SDO) and inhibition (i.e., IMS) did not predict stereotype-congruent responding $(\beta=-.011, S E=.01, z=-1.02, p=.30$, and $\beta=-.006$, $S E=.011, z=-.559, p=.54$, respectively). Taken together, these results suggest that learning decrements are not attributable to first-order stereotype application or inhibition occurring at the trial level (see Supplemental Table 13 in Supplemental for all model outputs). 
STEREOTYPES DISRUPT LEARNING

First order effects in the Athletic condition. Across all models, we likewise found no evidence that learning was disrupted on a trial-by-trial basis for participants in the Athletic condition. For instance, there was no difference in accuracy as a function of the race of the stimuli $(b=.01, S E=.04, z=.33, p=.73)$, no difference in accuracy or reaction time for stereotype-congruent versus incongruent trials $(b=.02, S E=.04, z=.51, p=.607$, and $b=$ $.001, S E=.006, t=-.245, p=.80$, respectively), nor were there any differences in the predictive weights of the card patterns for stereotype-congruent versus incongruent trials, regardless of whether card pattern weight was modeled as categorical (all $p$-values $>.25$ ) or as an absolute difference from $.5(b=-.01, S E=.01, z=-.709, p=.47)$. Moreover, individual differences associated with increased stereotype application (i.e., SDO) and inhibition (i.e., IMS) did not predict stereotype-congruent responding $(\beta=-.02, S E=.01, z=1.47, p=.14$, and $\beta=.01, S E=$ $.01, z=.78, p=.43$, respectively). Taken together, these results suggest that learning decrements are not attributable to first-order stereotype application or inhibition occurring at the trial level (see Supplemental Table 14 in Supplemental for all model outputs).

Second order effects in the Crime Face condition. If the presence of stereotypes does not disrupt learning on a trial-by-trial basis, an alternative explanation is that the presence of stereotypes is cognitively demanding which accumulates to disrupt learning. To test this, we examined whether internal or external motivations to respond without prejudice moderated (a) task performance, and (b) response times. Regarding performance, the first model revealed a significant main effect of IMS, and a significant Trial by IMS interaction $(\beta=-.13,95 \% C I=[-$ $.24,-.01], S E=.06, z=-2.23, O R=.87, p=.024$, and $\beta=-.06,95 \% C I=[-.12,-.01], S E=.027$, $z=-2.40, O R=.93, p=.016$, respectively), demonstrating that participants higher on IMS performed worse compared to those lower on IMS over time (see Figure 5). 
STEREOTYPES DISRUPT LEARNING

Regarding EMS, we observed a marginal trial by EMS interaction on accuracy $(\beta=-.05$, $95 \% C I=[-.10, .002], S E=.02, z=-1.87, O R=.95, p=.061)$, note however, the $95 \% C I$ for the interaction overlaps with 0 , and therefore this effect should be interpreted with caution.

Importantly, both the main effect of IMS and the IMS by trial interaction remained significant after controlling for EMS $(\beta=-.14,95 \% C I=[-.25,-.027], S E=.05, z=-2.44, O R=$ $.86, p=.014$, and $\beta=-.06,95 \% C I=[-.122,-.01], S E=.02, z=-2.49, O R=.93, p=.012$, respectively), whereas EMS was not significant after controlling for IMS $(\beta=.001, S E=.009, z$ $=.121, p=.90)$. We did not observe a significant IMS by EMS interaction $(\beta=-.02, S E=.048$, $z=-.62, p=.53)$, nor a significant three-way interaction between trial, IMS, and EMS ( $\beta=-$ $.009, S E=.023, z=-.41, p=.68)$.

Finally, examining the relationship between IMS and response times, as well as stimuli and response times for participants in the Crime Face condition revealed: (1) a significant trial by IMS interaction $(\beta=.02,95 \% C I=[.006, .0034], S E=.007, t=2.86, p=.004)$, suggesting that participants higher on IMS tend to have longer RTs over time, and (2) a significant main effect of stimuli $(\beta=-.009,95 \% C I=[-.018,-.0001], S E=.004, t=-1.99, p=.045)$, suggesting that participants tended to respond slower on trials when Black faces were present. Together, these findings help to bolster the claim that increased cognitive load for participants higher on IMS accumulates over the course of the task to disrupt learning (see Table 15 in Supplemental).

Second order effects in the Athletic condition. We did not find any evidence for second-order effects in the Athletic condition. For instance, both IMS and EMS were not associated with accuracy $(\beta=.03, S E=.08, z=.43, p=.662$, and $\beta=.03, S E=.08, z=.43, p=$ .665 , respectively). Likewise, both IMS and the race of the stimuli were not associated with 
STEREOTYPES DISRUPT LEARNING

response times $(\beta=.02, S E=.02, t=1.022, p=.31$, and $b=-.01, S E=.006, t=-.227, p=.11$, respectively: see Supplemental Table 16).

Taken together, these findings suggest that individuals who are more internally motivated to respond without prejudice learn significantly worse when learning in the presence of negatively-valenced stereotypes, even when controlling for EMS, whereas we found no such effect for participants in the positively-valenced stereotype condition.

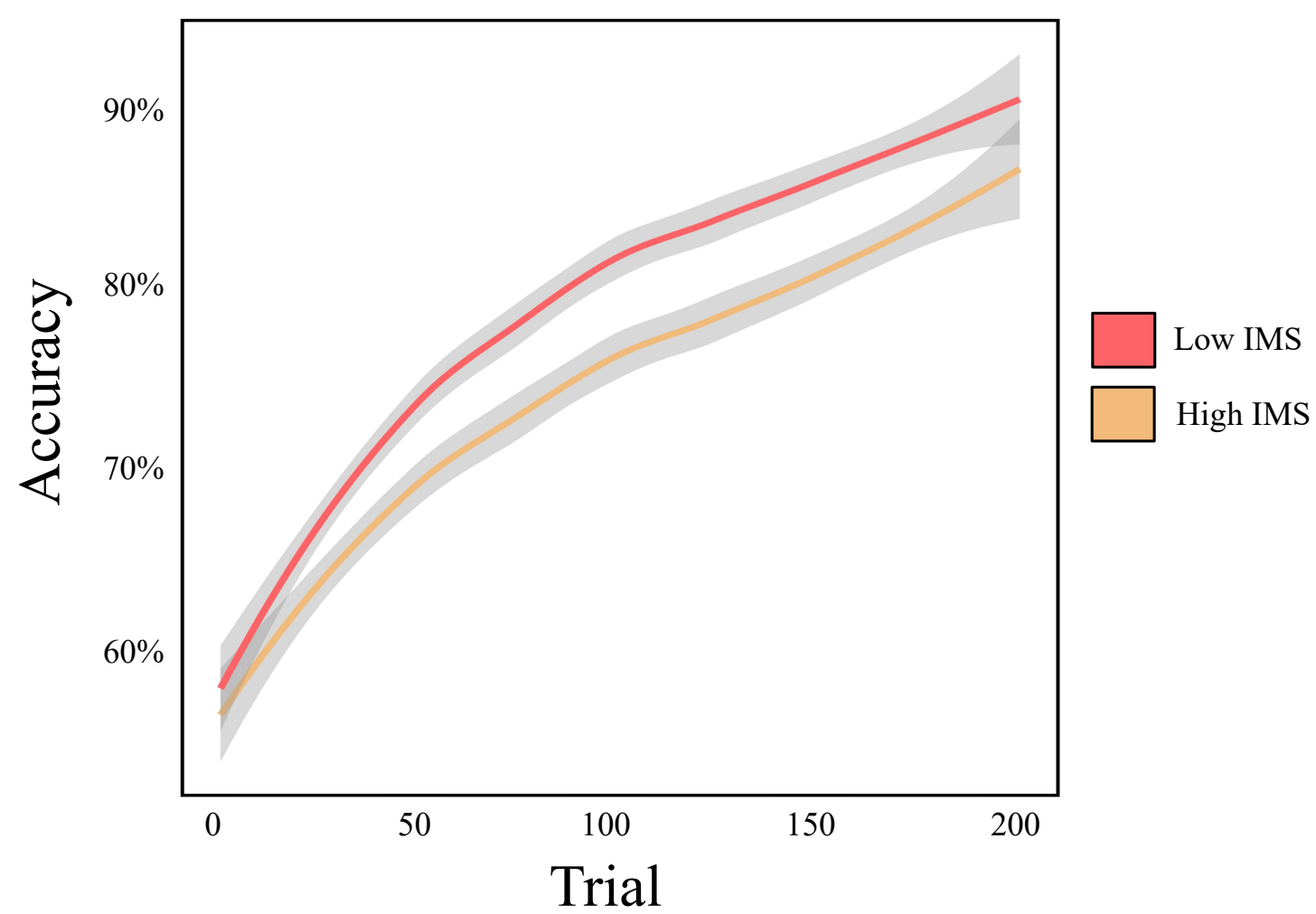

Figure 5. Learning rates (loess) as a function of low (red) and high (yellow) internal motivation to respond without prejudice. IMS was median split for visual purposes only. Note* that error bars denote standard error of the mean.

\section{Discussion}

These aggregated analyses tested competing hypotheses regarding whether learning decrements in the social conditions were attributable to first order effects due to stereotype 
STEREOTYPES DISRUPT LEARNING

application or inhibition, or whether learning was disrupted by second order effects due to increased cognitive load. Notably, although disruptions may occur via first or second order effects, these are not mutually exclusive. For instance, individuals high on internal motivations to respond without prejudice may suffer learning decrements due to increased cognitive load, but may also suffer learning decrements due to stereotype inhibition, as these motivations may manifest in increased cognitive load due self-monitoring of responses and/or increase stereotype inhibition.

A series of analyses that regressed both accuracy and choices onto different trial-level features (e.g., race of the face present, weights of the cards etc.) failed to provide evidence for first-order stereotype application or inhibition in either the Crime Face or Athletic conditions. That is, participants were no more or less likely to make trial by trial predictions that aligned or misaligned with the present stereotype. Instead, we find that individuals higher on internal motivation to respond without prejudice (IMS) suffered significantly worse learning decrements relative to those lower in the Crime Face condition. These results suggest that the presence of negative stereotypes are more salient for individuals with higher internal motivations to respond without prejudice as they activate egalitarian values and associated thoughts (Johns et al., 2008). As such, the presence of negative stereotypes may disrupt learning due to second order effects via increased cognitive load extraneous to the learning task. This is consistent with the assumption that the bottleneck for acquiring new knowledge is limited by working memory, and that cognitive effort extraneous to a learning task can disrupt optimal learning (Sweller, 2019).

Notably, IMS only moderated task performance for participants in the Crime Face condition but had no influence on learning in the Athletic condition, despite both environments leading to similar learning rates. This raises new questions about how pre-existing stereotypes 
STEREOTYPES DISRUPT LEARNING

disrupt learning, and whether positive and negative stereotypes disrupt learning via unique or parallel mechanisms. For instance, learning disruptions in the Athletic condition may also be attributable to cognitive load due to the presence of a sticky association. However, in this context where people are often less concerned about appearing prejudice, the disruption may instead be due to the attention required to follow task instructions and ignore the presence of a salient stereotype.

\section{General Discussion}

The current work extends decades of research on the destructive role of stereotypes on decision-making by examining whether and how they disrupt processes that precede decisionmaking, such as how people learn probabilistic associations in the first place. Across experiments, participants who were presented with task-irrelevant social associations (i.e., predicting crime in the presence of Black and White male faces) learned card pattern probabilities significantly worse than participant who were presented with task-irrelevant nonsocial associations (i.e., predicting weather in the presence of darker and lighter cloud formations), despite the fact that participants in both contexts were repeatedly told that the stimuli were irrelevant to the learning context and should therefore be ignored. This is remarkable considering that people maintain strong nonsocial associations throughout their life, such as associations between clouds and rain, just as they maintain strong social associations, such as stereotypes that connect race and crime.

These findings bear several important theoretical implications. First, probabilistic category learning is integral to how people learn about the world through trial and error (Dayan \& Niv, 2008; Gobet et al., 2001; Lee et al., 2012), yet extant research has not yet examined how pre-existing associations interfere with this learning process. As such, the current research 
STEREOTYPES DISRUPT LEARNING

interrogates a fundamental question about the boundary conditions of probabilistic category learning, demonstrating that pre-existing associations can interfere with new learning. Thus, the current work expands models of human learning by beginning to describe the cognitive processes by which pre-existing associations get in the way of new learning. These findings open up new avenues for future research on the different classes of pre-existing associations and their associated mechanisms that constrain learning, which we describe in the discussion below.

Second, different classes of pre-existing associations that people hold may impact new learning. For instance, people maintain both social (e.g., Black and criminal) and nonsocial (e.g., dark clouds with rain) pre-existing associations, but do they exert a similar influence over how people learn new associations? Our results suggest that there is something unique about preexisting social associations about race that disrupt learning, as these contexts led to significantly worse learning rates compared to a nonsocial context. Throughout daily life, people encounter many situations in which they must learn to predict discrete outcomes that are imperfectly correlated with predictive cues. Moreover, these situations are often embedded within contexts where task-irrelevant social and nonsocial cues compete for attention. These findings are the first to compare the effects of social versus nonsocial associations on new learning, and highlight the insidious nature of racial stereotypes by demonstrating that they disrupt the basic processes that people use to understand and make predictions about the world.

A third important implication of this work pertains to how stereotypes disrupt new category learning. The influence of stereotypes may be underpinned by at least two plausible mechanisms: first-order stereotype application or inhibition at the trial-level, and second-order cognitive load disruptions that accumulate across trials. We tested these competing hypotheses in a number of ways and failed to find any evidence for any first-order disruption effects. In other 
STEREOTYPES DISRUPT LEARNING

words, we found no evidence that task-irrelevant stereotypes increased or decreased the propensity for any particular stereotypically congruent or incongruent response in either the Crime Face or Athletic conditions. Instead, we find evidence that learning decrements in the Crime Face context were attributable to second-order effects, as people higher in IMS performed significantly worse than those lower in IMS. These results extend past research that finds that interracial interactions deplete cognitive resources that then disrupt performance on subsequent cognitive control tasks (Richeson et al., 2003). Specifically, we demonstrate that the cognitive demands of regulating responses that may appear prejudiced can disrupt learning as it unfolds in real time.

One potential explanation for why social associations disrupt learning via second-order effects is that they are generally more affect-laden (Zelazo \& Cunningham, 2007; Gawronski \& Bodenhausen, 2006; Stephan \& Stephan, 1985), and are therefore more salient during decisionmaking (Finucane et al., 2000; Todd et al., 2012). For instance, the presence of racial associations may elicit anticipated negative affect based on expected outcomes, such as people's fear of appearing prejudice (Mellers et al., 1997). One way to interrogate a purely affective mechanism is to examine whether learning decrements extend to nonsocial affective associations. For instance, people have prepared fear responses to certain classes of non-social stimuli (e.g., snakes, spiders; Öhman \& Soares, 1994), much like aversive learning that is facilitated by certain kinds of social stimuli that are perceived as threatening (e.g., Black faces; Olsson, 2005). If affect contributes to learning decrements, then people should also learn cueoutcome associations more poorly when they are paired with irrelevant affectively-laden nonsocial associations (e.g., predicting "bite" when spiders are present). Another way to test affect as a mechanism may be to measure arousal via indirect measures (e.g., eye-tracking, 
STEREOTYPES DISRUPT LEARNING

galvanic skin response; Proudfoot et al., 2016; Shi et al., 2007) during learning. In contrast to behavioral responses that people can monitor and control, people rarely have access or the ability to control their own physiological responses (Goff et al., 2008). Thus, indirect methods that measure arousal on a moment-to-moment basis may help to elucidate the conditions under which affective associations disrupt learning.

Consistent with the hypothesis that affect may moderate learning via second order effects due to anticipated negative affective responses, we find that participants with higher IMS suffered learning decrements more than those with lower IMS. Prior research has found that individuals higher on IMS are often more successful at regulating prejudicial responses (Plant \& Devine, 1998; Schlauch et al., 2009), and are less likely to show implicit forms of racial bias (e.g., associating negative words with Black faces and positive words with White faces; Devine et al., 2002). Conversely, people higher on EMS can successfully disguise prejudice on selfreport measures (Plant \& Devine, 1998), but often fail to inhibit more difficult to control racial bias (Butz \& Plant, 2009). One explanation for this discrepancy is that, whereas EMS tracks societal norms and obligations, IMS tracks personal values and goals which facilitate responses that are consistent with that value (Devine et al., 2002; Ryan \& Connell, 1989). The success of IMS in regulating bias is often driven by the negative feelings elicited when people violate their personal values (Plant \& Devine, 1998). Thus, despite explicit instructions that faces are not predictive of outcomes, participants higher on IMS may nevertheless experience negative arousal in anticipation that their responses may signal prejudice. As a result, individuals higher on IMS may monitor their responses, which increases extraneous cognitive effort and impedes learning.

The current findings also raise new questions about the influence of IMS on active versus passive learning contexts. The current findings suggest that participants higher on IMS are more 
STEREOTYPES DISRUPT LEARNING

sensitive to stereotypical cues when actively learning new probabilistic associations. At first blush, these findings diverge from research showing that participants higher on IMS are less sensitive to race during impression formation ( $\mathrm{Li}$ et al., 2016). One reason that our findings may diverge is that the tasks used in these studies are asking different things of participants. Specifically, the current work employs an active learning task in which participants make predictions and receive feedback in the presence of stereotype-eliciting faces that likely elicit conflict monitoring between task demands and internal goals to respond without prejudice (Dignath et al., 2020). Impression formation tasks differ from active learning tasks in that participants passively learn face/word pairs in the absence of predictions and feedback, and as such do not typically evoke the same responses (Mende-Siedlecki et al., 2013). Greater conflict monitoring for higher IMS individuals may tax cognitive functioning and disrupt learning in active versus passive learning tasks. These findings highlight a unique outcome of IMS previously not reported in the literature by demonstrating that IMS may inadvertently "get in the way" of optimal active learning as people attempt to monitor behaviors that may signal prejudice even when they are unrelated to the learning context.

A parallel explanation for learning decrements may be that social associations simply capture more attention and are therefore stickier and harder to ignore than nonsocial associations. For instance, we find no difference in learning between participants presented with positive stereotypes (e.g., Black and athletic) relative to participants presented with negative stereotypes (e.g., Black and criminal). Although motivations to respond without prejudice did not moderate learning rates in the positive stereotype condition, both positive and negative learning contexts led to similar task performance. This suggests that stereotypes might simply be stickier in general, regardless of affect. Indeed, research demonstrates that people are more likely to 
STEREOTYPES DISRUPT LEARNING

endorse stereotypes that they experience as less affectively-laden, such as stereotypes about gender and age (Czopp et al., 2015; Devine \& Elliot, 1995; Mae \& Carlston, 2005). This raises new questions about the role of affect and attention elicited by pre-existing associations in probabilistic learning, and whether they are context-dependent and dissociable based on the nature of the pre-existing associations. For instance, learning decrements caused by taskirrelevant positive associations may likewise be attributable to greater cognitive effort required to follow task instructions and ignore a salient association, regardless of whether it is affect-laden. One potential route to test whether such disruptions are due to the stickiness of social associations is to test whether this phenomenon generalizes to less affectively-charged social associations (e.g., status, gender, age). Doing so may shed light on whether specific learning mechanisms are context-dependent, such as anticipated negative affect for fear of social repercussion in one context versus the cognitive demand needed to ignore stereotypical associations in another.

The current findings also contribute to a growing body of research on the role of multiple memory systems in social cognition (Amodio, 2019). Converging evidence shows that humans have several types of memory systems (e.g., procedural, habitual, semantic, declarative) that are independently mediated by different brain systems, but that often work in parallel (Cabeza \& Moscovitch, 2013). The extent to which one system (e.g., declarative) is privileged over another (e.g., procedural) during learning can fluctuate and compete across time based on cognitive load (Foerde et al., 2006). For instance, prior research finds that participants employ different learning strategies when performing the WPT under stress (cold pressor) compared to control (Schwabe \& Wolf, 2013). The stress manipulation modulated the engagement of different memory system, such that stressed participants used less declarative (i.e., hippocampus-based) and more 
STEREOTYPES DISRUPT LEARNING

procedural (i.e., striatum-based) learning systems compared to control. This shift towards procedural learning under stress also rescued task performance, but disrupted task performance when participants tried to engage declarative learning (i.e., explicitly learning the card probabilities). The notion that multiple memory systems simultaneously mediate learning may help to explain how second-order effects disrupt learning. For instance, analogous to the influence of stress on learning, the presence of stereotype may engage more procedural learning mechanisms. However, the deliberate act of self-monitoring responses may engage declarative learning, thereby suppressing optimal learning (Aggregated Analysis). While participants may overcome this competition later in the task, the suppressed learning rates early on may accumulate to disrupt overall learning. Future research should explicitly test whether social versus nonsocial learning contexts engage different learning mechanism and associated memory systems.

\section{Limitations}

The current findings and their implications should be considered within the limitations of each study. For instance, we did not include a pure control condition where participants learned in the absence of any additional stimuli. Future research that includes a pure control condition would help to rule out alternative explanations, such as whether the presence of clouds improved performance or the presence of faces reduced it. However, it is highly unlikely that performance would be improved in the presence of clouds given that the stimuli were orthogonal to the learning task and thus could not improve performance. Moreover, Study 2 was designed to rule out a number of alternative explanations by employing a fully crossed design that controlled for context and stimulus-driven effects. For instance, if the effects are driven by the forecasting context, whereby one kind of prediction (e.g., crime) is stranger than the other (e.g., weather), 
STEREOTYPES DISRUPT LEARNING

then we would expect a significant difference between participants predicting crime in the presence of clouds from participants predicting weather in the presence of faces, which we did not find. Likewise, if the effects are drive by the stimuli (i.e., the presence of faces or clouds) then we would expect a significant difference between the Weather Cloud and Weather Face condition, which we also did not find. Our goal in the current research was to directly compare the influence of social to nonsocial pre-existing associations on new learning to determine which exerts a stronger influence on the learning context. A pure control condition would help to shed further light on whether learning in the presence of nonsocial pre-existing associations disrupts performance compared to learning in the absence of any pre-existing associations.

Relatedly, the current findings are limited to contexts involving pre-existing associations about the weather and race, and as such we cannot generalize across all social and nonsocial learning contexts. An alternative explanation is that learning decrements are attributable to social learning environments where faces are present, even when they are devoid of stereotypical associations. For instance, it may be that social associations are simply more sticky than nonsocial associations about weather, and that these learning decrements would persist in a context where participants make social forecasts (e.g., "fired" or "not fired" from a job) in the presence of White and Black faces. The goal of the current work was to test whether one class of distractors-such as racial associations-are more disruptive than other distractors, rather than to demonstrate all different contexts under which these effects occur. We believe that the current findings are generative and invite future research to examine the interplay between different preexisting associations and new learning contexts.

Another potential limitation of the current research is that it is unclear whether preexisting associations disrupt the acquisition of new learning or rather people's performance on 
STEREOTYPES DISRUPT LEARNING

the task. In other words, because learning takes place gradually over time, it is unclear if taxed cognitive functioning impairs people's ability to learn the new associations or merely their ability to produce the learned skill and make the correct response. Past work suggests that performance-rather than learning-may be disrupted during a probabilistic task when accompanied by a secondary cognitive task (Foerde et al., 2007). However, in this past work, participants were asked to optimize performance on both cognitive tasks simultaneously, whereas participants in the current work were explicitly told to ignore any distractors and instead focus on the card or card patterns. More research is needed to test the extent to which taskirrelevant associations interfere with the acquisition or expression of newly learned probabilistic associations.

\section{Conclusions}

People constantly learn new associations by making iterative predictions and observing feedback in order to navigate their environments successfully. An important challenge to the learning process is learning when to ignore task-irrelevant prior associations that may 'get in the way' of optimal learning. The current findings highlight the insidious and sticky nature of stereotypes on learning: Even when stereotypic associations are explicitly task-irrelevant, they influence how people learn, think, and behave. In order to achieve sustainable social progress, we need to better understand how stereotypes affect the basic processes we use for understanding and making predictions about the world, to pinpoint the exact mechanisms and contexts in which they operate. The current work is a step in this direction.

\section{Context of the Research}

Our research group with members from San Francisco State University and the University of California, Riverside investigated whether associations of a social nature may be particularly 
STEREOTYPES DISRUPT LEARNING

difficult to ignore, and thus impinge on learning more than nonsocial associations. Building on decades of research on stereotyping and prejudice, we provide evidence that the mere presence of stereotypes disrupts probabilistic learning and updating even when they are orthogonal to task demands. In future work we aim to generalize these findings across different learning environments. 
STEREOTYPES DISRUPT LEARNING

\section{References}

Allidina, S., \& Cunningham, W. A. (2021). Avoidance begets avoidance: A computational account of negative stereotype persistence. Journal of Experimental Psychology: General, https://doi.org/10.1037/xge0001037

Amodio, D. M. (2019). Social Cognition 2.0: An Interactive Memory Systems Account. Trends in Cognitive Sciences, 23(1), 21-33. https://doi.org/10.1016/j.tics.2018.10.002

Arrow, K. J. (1998). What Has Economics to Say About Racial Discrimination? Journal of Economic Perspectives, 12(2), 91-100. https://doi.org/10.1257/jep.12.2.91

Ashton, M. C., \& Lee, K. (2007). Empirical, Theoretical, and Practical Advantages of the HEXACO Model of Personality Structure. Personality and Social Psychology Review, 11(2), 150-166. https://doi.org/10.1177/1088868306294907

Ashton, M. C., Lee, K., \& de Vries, R. E. (2014). The HEXACO Honesty-Humility, Agreeableness, and Emotionality Factors: A Review of Research and Theory. Personality and Social Psychology Review, 18(2), 139-152. https://doi.org/10.1177/1088868314523838

Axt, J. R., Ebersole, C. R., \& Nosek, B. A. (2014). The Rules of Implicit Evaluation by Race, Religion, and Age. Psychological Science. https://doi.org/10.1177/0956797614543801

Bates, D., Mächler, M., Bolker, B., \& Walker, S. (2015). Fitting Linear Mixed-Effects Models Using lme4. Journal of Statistical Software, 67(1), 1 - 48. doi:http://dx.doi.org/10.18637/jss.v067.i01 
STEREOTYPES DISRUPT LEARNING

Barnett, B. O., Brooks, J. A., \& Freeman, J. B. (2021). Stereotypes bias face perception via orbitofrontal-fusiform cortical interaction. Social Cognitive and Affective Neuroscience, 16(3), 302-314. https://doi.org/10.1093/scan/nsaa165

Bergsieker, H. B., Leslie, L. M., Constantine, V. S., \& Fiske, S. T. (2012). Stereotyping by omission: Eliminate the negative, accentuate the positive. Journal of Personality and Social Psychology, 102(6), 1214-1238. https://doi.org/10.1037/a0027717

Blair, I., Judd, C. M., \& Chapleau, K. M. (2004). The Influence of Afrocentric Facial Features in Criminal Sentencing. Psychological Science, 15(10), 674-679. https://doi.org/10.1111/j.0956-7976.2004.00739.x

Blair, M., \& Homa, D. L. (2005). Integrating Novel Dimensions to Eliminate Category Exceptions: When More Is Less. Journal of Experimental Psychology: Learning, Memory, and Cognition, 31(2), 258-271. https://doi.org/10.1037/0278-7393.31.2.258

Blind, G. D., \& Lottanti von Mandach, S. (2021). Of pride and prejudice: Agent learning under sticky and persistent stereotype. Journal of Economic Interaction and Coordination, 16(2), 381-410. https://doi.org/10.1007/s11403-020-00307-0

Blinder, S., Ford, R. and Ivarsflaten, E. (2013), The Better Angels of Our Nature: How the Antiprejudice Norm Affects Policy and Party Preferences in Great Britain and Germany. American Journal of Political Science, 57: 841-857. https://doi.org/10.1111/ajps.12030

Bodenhausen, G. V., \& Wyer, R. S., Jr (1985). Effects of stereotypes on decision making and information-processing strategies. Journal of personality and social psychology, 48(2), 267-282. https://doi.org.0.1037/0022-3514.48.2.267

Brewer, M. B. (2001). The Many Faces of Social Identity: Implications for Political Psychology. Political Psychology, 22(1), 115-125. https://doi.org/10.1111/0162-895x.00229 
STEREOTYPES DISRUPT LEARNING

Brewer, M. B., \& Pierce, K. P. (2005). Social identity complexity and outgroup tolerance. Personality and Social Psychology Bulletin, 31(3), 428-437. https://doi.org/10.1177/0146167204271710

Bürkner, P.C. (2017). brms: An R Package for Bayesian Multilevel Models Using Stan. Journal of Statistical Software, 80(1). https://doi.org/10.18637/jss.v080.i01

Butz, D. A., \& Plant, E. A. (2009). Prejudice control and interracial relations: the role of motivation to respond without prejudice. Journal of personality, 77(5), 1311-1341. https://doi.org/10.1111/j.1467-6494.2009.00583.x

Cabeza, R., \& Moscovitch, M. (2013). Memory Systems, Processing Modes, and Components: Functional Neuroimaging Evidence. Perspectives on Psychological Science, 8(1), 49-55. https://doi.org/10.1177/1745691612469033

Chang, L. W., Krosch, A. R., \& Cikara, M. (2016). Effects of intergroup threat on mind, brain, and behavior. Current Opinion in Psychology, 11, 69-73. https://doi.org/10.1016/j.copsyc.2016.06.004

Cleeremans, A., \& McClelland, J. L. (1991). Learning the structure of event sequences. Journal of Experimental Psychology: General, 120(3), 235-253. https://doi.org/10.1037/00963445.120.3.235. 19 .

Correll, J., Park, B., Judd, C. M., \& Wittenbrink, B. (2002). The police officer's dilemma: Using ethnicity to disambiguate potentially threatening individuals. Journal of Personality and Social Psychology, 83(6), 1314-1329. https://doi.org/10.1037/0022-3514.83.6.1314

Correll, J., Wittenbrink, B., Crawford, M. T., \& Sadler, M. S. (2015). Stereotypic vision: How stereotypes disambiguate visual stimuli. Journal of Personality and Social Psychology, 108(2), 219-233. https://doi.org/10.1037/pspa0000015 
STEREOTYPES DISRUPT LEARNING

Craig, S., Lewandowsky, S., \& Little, D. R. (2011). Error discounting in probabilistic category learning. Journal of Experimental Psychology: Learning, Memory, and Cognition, 37(3), 673-687. https://doi.org/10.1037/a0022473

Cunningham, W. A., \& Zelazo, P. D. (2007). Attitudes and evaluations: A social cognitive neuroscience perspective. Trends in Cognitive Sciences, 11(3), 97-104. https://doi.org/10.1016/j.tics.2006.12.005

Czopp, A. M., Kay, A. C., \& Cheryan, S. (2015). Positive Stereotypes Are Pervasive and Powerful. Perspectives on Psychological Science, 10(4), 451-463. https://doi.org/10.1177/1745691615588091

Czopp, A. M. (2008). When is a compliment not a compliment? Evaluating expressions of positive stereotypes. Journal of Experimental Social Psychology, 44(2), 413-420. https://doi.org/10.1016/j.jesp.2006.12.007

Dayan P, Niv Y. (2008). Reinforcement learning: the good, the bad and the ugly. Curr Opin Neurobiol. 18(2):185-96. https://doi.org/10.1016/j.conb.2008.08.003

Daw, N. D., O’Doherty, J. P., Dayan, P., Seymour, B., \& Dolan, R. J. (2006). Cortical substrates for exploratory decisions in humans. Nature, 441(7095), 876-879. https://doi.org/10.1038/nature04766

Derreumaux, Y. (2022). data and analyses for the manuscript entitled stereotypes disrupt probabilistic learning. Retrieved from osf.io/fxkh7

Devine, P. G. (1989). Stereotypes and prejudice: Their automatic and controlled components. Journal of Personality and Social Psychology, 56(1), 5-18. doi:http://dx.doi.org/10.1037/0022-3514.56.1.5 
STEREOTYPES DISRUPT LEARNING

Devine, P. G., \& Elliot, A. J. (1995). Are Racial Stereotypes Really Fading? The Princeton Trilogy Revisited. Personality and Social Psychology Bulletin, 21(11), 1139-

\section{0. https://doi.org/10.1177/01461672952111002}

Devine, P., Plant, E. A., Amodio, D. M., Harmon-Jones, E., \& Vance, S. L. (2002). The regulation of explicit and implicit race bias: The role of motivations to respond without prejudice. Journal of Personality and Social Psychology, 82(5), 835-848. https://doi.org/10.1037/0022-3514.82.5.835

Dignath, D., Eder, A. B., Steinhauser, M., \& Kiesel, A. (2020). Conflict monitoring and the affective-signaling hypothesis-An integrative review. Psychonomic Bulletin \& Review, 27(2), 193-216. https://doi.org/10.3758/s13423-019-01668-9

Eberhardt, J. L., Goff, P. A., Purdie, V. J., \& Davies, P. G. (2004). Seeing Black: Race, Crime, and Visual Processing. Journal of Personality and Social Psychology, 87(6), 876-893. https://doi.org/10.1037/0022-3514.87.6.876

Eisenhauer, J., (2021). Meta-analysis and mega-analysis: A simple introduction. Teaching Statistics, 43(1), 21-27. https://doi.org/10.1111/test.12242

Finucane, M.L., Alhakami, A., Slovic, P. and Johnson, S.M. (2000). The affect heuristic in judgments of risks and benefits. Journal of Behavioral Decision Making, 13: 1-

17. https://doi.org/10.1002/(SICI)1099-0771(200001/03)13:1<1::AIDBDM333>3.0.CO;2-S

Fiske, S. T., \& Neuberg, S. L. (1990). A Continuum of Impression Formation, from Category Based to Individuating Processes: Influences of Information and Motivation on Attention and Interpretation. In Advances in Experimental Social Psychology (Vol. 23, pp. 1-74). Elsevier. https://doi.org/10.1016/S0065-2601(08)60317-2 
STEREOTYPES DISRUPT LEARNING

Foerde, K., Knowlton, B. J., \& Poldrack, R. A. (2006). Modulation of competing memory systems by distraction. Proceedings of the National Academy of Sciences, 103(31), 11778-11783. https://doi.org/10.1073/pnas.0602659103

Foerde, K., Poldrack, R. A., \& Knowlton, B. J. (2007). Secondary-task effects on classification learning. Memory \& Cognition, 35(5), 864-874. https://doi.org/10.3758/BF03193461

Frank, M. C., Vul, E., \& Johnson, S. P. (2009). Development of infants' attention to faces during the first year. Cognition, 110(2), 160-170. https://doi.org/10.1016/j.cognition.2008.11.010

Gawronski, B., \& Bodenhausen, G. V. (2006). Associative and propositional processes in evaluation: An integrative review of implicit and explicit attitude change. Psychological Bulletin, 132(5), 692-731. https://doi.org/10.1037/0033-2909.132.5.692

Gluck, M. A. (2002). How do People Solve the "Weather Prediction" Task?: Individual Variability in Strategies for Probabilistic Category Learning. Learning \& Memory, 9(6), 408-418. https://doi.org/10.1101/1m.45202

Gobet, F., Lane, P., Croker, S., Cheng, P., Jones, G., Oliver, I., \& Pine, J. (2001). Chunking mechanisms in human learning. Trends in Cognitive Sciences, 5(6), 236-243. https://doi.org/10.1016/S1364-6613(00)01662-4

Goff, P. A., Eberhardt, J. L., Williams, M. J., \& Jackson, M. C. (2008). Not yet human: Implicit knowledge, historical dehumanization, and contemporary consequences. Journal of Personality and Social Psychology, 94(2), 292-306. https://doi.org/10.1037/0022$\underline{3514.94 .2 .292}$

Goh, J. X., Hall, J. A., \& Rosenthal, R. (2016). Mini Meta-Analysis of Your Own Studies: Some Arguments on Why and a Primer on How. Social and Personality Psychology Compass, 
STEREOTYPES DISRUPT LEARNING

10(10), 535-549. https://doi.org/10.1111/spc3.12267

Green, P., \& Macleod, C. J. (2016). SIMR: An R package for power analysis of generalized linear mixed models by simulation. Methods in Ecology and Evolution, 7(4), 493-498. https://doi.org/10.1111/2041-210X.12504

Hogg, Michael \& Terry, Deborah \& White, Katherine. (1995). A Tale of Two Theories: A Critical Comparison of Identity Theory with Social Identity Theory. Social Psychology Quarterly. 58. 10.2307/2787127.

Islam, M. R., \& Hewstone, M. (1993). Dimensions of Contact as Predictors of Intergroup Anxiety, Perceived Out-Group Variability, and Out-Group Attitude: An Integrative Model. Personality and Social Psychology Bulletin, 19(6), 700-710. https://doi.org/10.1177/0146167293196005

Johns, M., Cullum, J., Smith, T., \& Freng, S. (2008). Internal motivation to respond without prejudice and automatic egalitarian goal activation. Journal of Experimental Social Psychology, 44(6), 1514-1519. https://doi.org/10.1016/j.jesp.2008.07.003

Kay, A. C., Day, M. V., Zanna, M. P., \& Nussbaum, A. D. (2013). The insidious (and ironic) effects of positive stereotypes. Journal of Experimental Social Psychology, 49(2), 287291. https://doi.org/10.1016/j.jesp.2012.11.003

Kirsch, I., Lynn, S. J., Vigorito, M., \& Miller, R. R. (2004). The role of cognition in classical and operant conditioning. Journal of Clinical Psychology, 60(4), 369-392. https://doi.org/10.1002/jclp.10251

Knowlton, B. J., Squire, L. R., \& Gluck, M. A. (1994). Probabilistic classification learning in amnesia. Learning \& memory, 1(2), 106-120. 
STEREOTYPES DISRUPT LEARNING

Krieglmeyer, R., \& Sherman, J. W. (2012). Disentangling stereotype activation and stereotype application in the stereotype misperception task. Journal of Personality and Social Psychology, 103(2), 205-224. https://doi.org/10.1037/a0028764

Kruschke, J. K. (2010). Bayesian data analysis. WIREs Cognitive Science, 1(5), 658-676. https://doi.org/10.1002/wcs.72

Kruschke, J. K. (2018). Rejecting or Accepting Parameter Values in Bayesian Estimation. Advances in Methods and Practices in Psychological Science, 270-280. https://doi.org/10.1177/2515245918771304

Kruschke, J. K., \& Johansen, M. K. (1999). A Model of Probabilistic Category Learning. Journal of Experimental Psychology: Learning, Memory, and Cognition, 37. 25(5), 1083-1119. https://doi.org/10.1037//0278-7393.25.5.1083

Kruschke, J. K., \& Liddell, T. M. (2018). Bayesian data analysis for newcomers. Psychonomic Bulletin \& Review, 25(1), 155-177. https://doi.org/10.3758/s13423-017-1272-1

Kteily, N., Ho, A. K., \& Sidanius, J. (2012). Hierarchy in the mind: The predictive power of social dominance orientation across social contexts and domains. Journal of Experimental Social Psychology, 48(2), 543-549. https://doi.org/10.1016/j.jesp.2011.11.007

Kumaran, D., Hassabis, D., \& McClelland, J. L. (2016). What Learning Systems do Intelligent Agents Need? Complementary Learning Systems Theory Updated. Trends in Cognitive Sciences, 20(7), 512-534. https://doi.org/10.1016/j.tics.2016.05.004

Kurdi, B., Mann, T. C., Charlesworth, T. E. S., \& Banaji, M. R. (2019). The relationship between implicit intergroup attitudes and beliefs. Proceedings of the National Academy of Sciences, 116(13), 5862-5871. https://doi.org/10.1073/pnas.1820240116 
STEREOTYPES DISRUPT LEARNING

Kuznetsova, A., Brockhoff, P. B., \& Christensen, R. H. B. (2017). lmerTest Package: Tests in Linear Mixed Effects Models. Journal of Statistical Software, 82(13). https://doi.org/10.18637/jss.v082.i13

Lee, D., Seo, H., \& Jung, M. W. (2012). Neural Basis of Reinforcement Learning and Decision Making. Annual Review of Neuroscience, 35(1), 287-308. https://doi.org/10.1146/annurev-neuro-062111-150512

Li, T., Cardenas-Iniguez, C., Correll, J., \& Cloutier, J. (2016). The impact of motivation on racebased impression formation. NeuroImage, 124(Pt A), 1-7. https://doi.org/10.1016/j.neuroimage.2015.08.035

Ma, D. S., Correll, J., \& Wittenbrink, B. (2015). The Chicago face database: A free stimulus set of faces and norming data. Behavior Research Methods, 47(4), 1122-1135. https://doi.org/10.3758/s13428-014-0532-5

Mae, L., \& Carlston, D. E. (2005). Hoist on your own petard: When prejudiced remarks are recognized and backfire on speakers. Journal of Experimental Social Psychology, 41(3), 240-255. https://doi.org/10.1016/j.jesp.2004.06.011

Mellers, B. A., Schwartz, A., Ho, K., \& Ritov, I. (1997). Decision affect theory: Emotional reactions to the outcomes of risky options. Psychological Science, 8(6), 423-429. https://doi.org/10.1111/j.1467-9280.1997.tb00455.x

Mende-Siedlecki, P., Cai, Y., \& Todorov, A. (2013). The neural dynamics of updating person impressions. Social cognitive and affective neuroscience, 8(6), 623-631. https://doi.org/10.1093/scan/nss040

Minhas, R., \& Walsh, D. (2021). The role of prejudicial stereotypes in the formation of suspicion: An examination of operational procedures in stop and search practices. 
STEREOTYPES DISRUPT LEARNING

International Journal of Police Science \& Management, 23(3), 293-305.

https://doi.org/10.1177/14613557211016499

Niv, Y., Daniel, R., Geana, A., Gershman, S. J., Leong, Y. C., Radulescu, A., \& Wilson, R. C.

(2015). Reinforcement Learning in Multidimensional Environments Relies on Attention Mechanisms. Journal of Neuroscience, 35(21), 8145-8157.

https://doi.org/10.1523/JNEUROSCI.2978-14.2015

Öhman, A., \& Soares, J. J. F. (1994). “Unconscious anxiety”: Phobic responses to masked stimuli. Journal of Abnormal Psychology, 103(2), 231-240.

https://doi.org/10.1037/0021-843X.103.2.231

Olsson, A. (2005). The Role of Social Groups in the Persistence of Learned Fear. Science, 309(5735), 785-787. https://doi.org/10.1126/science.1113551

Onorato, R., \& Turner, J. (2004). Fluidity in the self-concept: The shift from personal to social identity. European Journal of Social Psychology, 34(3), 257-278.

https://doi.org/10.1002/ejsp.195

Ozer, D. J., \& Benet-Martínez, V. (2006). Personality and the prediction of consequential outcomes. Annual Review of Psychology, 57, 401-421.

https://doi.org/10.1146/annurev.psych.57.102904.190127

Paas, F., \& van Merriënboer, J. J. G. (2020). Cognitive-Load Theory: Methods to Manage Working Memory Load in the Learning of Complex Tasks. Current Directions in Psychological Science, 29(4), 394-398. https://doi.org/10.1177/0963721420922183

Paulhus, D. L. (1984). Two-Component Models of Socially Desirable Responding. Journal of Personality and Social Psychology, 12. https://doi.org/10.1037/0022-3514.46.3.598

Payne, B. K. (2001). Prejudice and perception: The role of automatic and controlled processes in misperceiving a weapon. Journal of Personality and Social Psychology, 81(2), 181-192. 
STEREOTYPES DISRUPT LEARNING

https://doi.org/10.1037/0022-3514.81.2.181

Pearson, A. R., Dovidio, J. F., \& Gaertner, S. L. (2009). The Nature of Contemporary Prejudice: Insights from Aversive Racism: Contemporary Prejudice. Social and Personality Psychology Compass, 3(3), 314-338. https://doi.org/10.1111/j.1751-9004.2009.00183.x

Plant, Ashby \& Devine, Patricia. (1998). Internal and External Motivation to Respond Without Prejudice. Journal of Personality and Social Psychology. 75. 811-832. 10.1037/00223514.75.3.811.

Pratto, F., Sidanius, J., Stallworth, L. M., \& Malle, B. F. (1994). Social dominance orientation: A personality variable predicting social and political attitudes. Journal of Personality and Social Psychology, 67(4), 741-763. https://doi.org/10.1037/0022-3514.67.4.741

Proudfoot, J. G., Jenkins, J. L., Burgoon, J. K., \& Nunamaker, J. F. (2016). More Than Meets the Eye: How Oculometric Behaviors Evolve Over the Course of Automated Deception Detection Interactions. Journal of Management Information Systems, 33(2), 332-360. https://doi.org/10.1080/07421222.2016.1205929

Rand, D. G. (2012). The promise of Mechanical Turk: How online labor markets can help theorists run behavioral experiments. Journal of Theoretical Biology, 299, 172-179. https://doi.org/10.1016/j.jtbi.2011.03.004

Rescorla, R., \& Wagner, Allan. R. (1972). A theory of Pavlovian conditioning: Variations in the effectiveness of reinforcement and nonreinforcement. In Classical conditioning: Current research and theory. Appleton-Century-Crofts.

Richeson, J. A., Baird, A. A., Gordon, H. L., Heatherton, T. F., Wyland, C. L., Trawalter, S., \& Shelton, J. N. (2003). An fMRI investigation of the impact of interracial contact on 
STEREOTYPES DISRUPT LEARNING

executive function. Nature Neuroscience, 6(12), 1323-1328.

https://doi.org/10.1038/nn1156

Richeson, J. A., \& Shelton, J. N. (2003). When Prejudice Does Not Pay: Effects of Interracial Contact on Executive Function. Psychological Science, 14(3), 287-290. https://doi.org/10.1111/1467-9280.03437

Richeson, J. A., \& Trawalter, S. (2005). Why do interracial interactions impair executive function? A resource depletion account. Journal of Personality and Social Psychology, 88(6), 934-947. https://doi.org/10.1037/0022-3514.88.6.934

Rubien-Thomas, E., Berrian, N., Cervera, A., Nardos, B., Cohen, A. O., Lowrey, A., Daumeyer, N. M., Camp, N. P., Hughes, B. L., Eberhardt, J. L., Taylor-Thompson, K. A., Fair, D. A., Richeson, J. A., \& Casey, B. J. (2021). Processing of Task-Irrelevant Race Information is Associated with Diminished Cognitive Control in Black and White Individuals. Cognitive, Affective, \& Behavioral Neuroscience, 21(3), 625-638. https://doi.org/10.3758/s13415-021-00896-8

Rumelhart, D., Hinton, G., \& Mcclelland, J. (1986). A General Framework for Parallel Distributed Processing. In Parallel distributed processing: Explorations in the microstructure of cognition. MIT Press.

Ryan, R. M., \& Connell, J. P. (1989). Perceived locus of causality and internalization: examining reasons for acting in two domains. Journal of personality and social psychology, 57(5), 749-761. https://doi.org/10.1037//0022-3514.57.5.749

Simon H. A. (1956). Rational choice and the structure of the environment. Psychological review, 63(2), 129-138. https://doi.org/10.1037/h0042769 
STEREOTYPES DISRUPT LEARNING

Siy, J. O., \& Cheryan, S. (2016). Prejudice Masquerading as Praise: The Negative Echo of Positive Stereotypes. Personality and Social Psychology Bulletin, 42(7), 941-954. https://doi.org/10.1177/0146167216649605

Schiller, D., Freeman, J. B., Mitchell, J. P., Uleman, J. S., \& Phelps, E. A. (2009). A neural mechanism of first impressions. Nature Neuroscience, 12(4), 508-514. https://doi.org/10.1038/nn.2278

Schlauch, R. C., Lang, A. R., Plant, E. A., Christensen, R., \& Donohue, K. F. (2009). Effect of alcohol on race-biased responding: The moderating role of internal and external motivations to respond without prejudice. Journal of Studies on Alcohol and Drugs, 70(3), 328-336. https://doi.org/10.15288/jsad.2009.70.328

Schwabe, L., \& Wolf, O. T. (2013). Stress and multiple memory systems: From 'thinking' to ‘doing.' Trends in Cognitive Sciences, 17(2), 60-68. https://doi.org/10.1016/j.tics.2012.12.001

Shelton, J. N., \& Richeson, J. A. (2005). Intergroup Contact and Pluralistic Ignorance. Journal of Personality and Social Psychology, 88(1), 91-107. https://doi.org/10.1037/00223514.88.1.91

Shi, Y., Ruiz, N., Taib, R., Choi, E., \& Chen, F. (2007). Galvanic skin response (GSR) as an index of cognitive load. CHI '07 Extended Abstracts on Human Factors in Computing Systems, 2651. https://doi.org/10.1145/1240866.1241057

Soto C. J., John O. P. (2017). The next Big Five Inventory (BFI-2): Developing and assessing a hierarchical model with 15 facets to enhance bandwidth, fidelity, and predictive power. $J$ Pers Soc Psychol. 113(1):117-143. https://doi.org/10.1037/pspp0000096.

Staddon, J. E. R., \& Cerutti, D. T. (2003). Operant Conditioning. Annual Review of Psychology, 
STEREOTYPES DISRUPT LEARNING

54(1), 115-144. https://doi.org/10.1146/annurev.psych.54.101601.145124

Stephan, W.G. and Stephan, C.W. (1985), Intergroup Anxiety. Journal of Social Issues, 41: $157-$ 175. https://doi.org/10.1111/j.1540-4560.1985.tb01134.x

Suri, G., Gross, J. J., \& McClelland, J. L. (2020). Value-based decision making: An interactive activation perspective. Psychological review, 127(2), 153-185.

https://doi.org/10.1037/rev0000164

Sweller, J. (2010). Element Interactivity and Intrinsic, Extraneous, and Germane Cognitive Load. Educational Psychology Review 22, 123-138. https://doi.org/10.1007/s10648-010$9128-5$

Sweller, J. (2011). Cognitive Load Theory. In Psychology of Learning and Motivation Advances in Research and Theory (Vol. 55). Elsevier Inc. https://doi.org/10.1016/B978$0-12-387691-1.00002-8$

Sweller, J., van Merriënboer, J. J. G., \& Paas, F. (2019). Cognitive Architecture and Instructional Design: 20 Years Later. Educational Psychology Review, 31(2), 261-292. https://doi.org/10.1007/s10648-019-09465-5

Tausch, N., Hewstone, M., Kenworthy, J. B., Psaltis, C., Schmid, K., Popan, J. R., Cairns, E., \& Hughes, J. (2010). Secondary transfer effects of intergroup contact: Alternative accounts and underlying processes. Journal of Personality and Social Psychology, 99(2), 282302. https://doi.org/10.1037/a0018553

Theeuwes, J., \& Van der Stigchel, S. (2006). Faces capture attention: Evidence from inhibition of return. Visual Cognition, 13(6), 657-665.https://doi.org/10.1080/13506280500410949 


\section{STEREOTYPES DISRUPT LEARNING}

Todd, R. M., Cunningham, W. A., Anderson, A. K., \& Thompson, E. (2012). Affect-biased attention as emotion regulation. Trends in Cognitive Sciences, 16(7), 365-372. https://doi.org/10.1016/j.tics.2012.06.003

Todorov, A., \& Mende-Siedlecki, P. (2014). The cognitive and neural basis of impression formation. In The Oxford handbook of cognitive neuroscience, Vol. 2: The cutting edges. (pp. 188-203). Oxford University Press.

Trawalter, S., Todd, A. R., Baird, A. A., \& Richeson, J. A. (2008). Attending to threat: Race based patterns of selective attention. Journal of Experimental Social Psychology, 44(5), 1322-1327. https://doi.org/10.1016/j.jesp.2008.03.006

Welch, K. (2007). Black Criminal Stereotypes and Racial Profiling. Journal of Contemporary Criminal Justice, 23(3), 276-288. https://doi.org/10.1177/1043986207306870

Willer, D., Turner, J. C., Hogg, M. A., Oakes, P. J., Reicher, S. D., \& Wetherell, M. S. (1989). Rediscovering the Social Group: A Self-Categorization Theory. In Contemporary Sociology (Vol. 18, Issue 4, p. 645). https://doi.org/10.2307/2073157

Zelazo \& Cunningham, W., P. D. (2007). Executive function: Mechanisms underlying emotion regulation. Handbook of Emotion Regulation, 12(11), 135-158.

http://dx.doi.org/10.1038/nrn3119\%5Cnhttp://www.scopus.com/inward/record.url?eid=2 s2.077953191733\&partnerID=tZOtx3y1\%5Cnhttp://bmcneurosci.biomedcentral.com/arti cles/10.1186/1471-2202-12-2 
STEREOTYPES DISRUPT LEARNING

\section{Supplementary Materials for}

\section{Stereotypes Disrupt Probabilistic Category Learning}

This document includes:

Supplementary Materials and Methods

Supplementary Analyses and Regression Tables 


\section{Supplementary Materials and Methods}

\section{Task Creation (description of how JavaScript paradigm was created)}

The paradigm was designed and implemented using the following design patterns and techniques.

To begin designing the application we broke the trials down into separate objects, each representing a stimuli or function of the trial. This process yielded two classes not including the Main class which will run the trial itself. Those two classes were Card, and Pattern.

The card class contains a variable which stores what type of card it is. That is the only function of the card class.

The pattern class contains an array of Cards, as well as variables to store pattern frequency, pattern weights (to steal, not to steal), and the non-related pattern outcome (they did steal, they didn't steal).

The main class implements the Pattern class, passing in the 14 unique pattern variations to the Patterns constructor. After instantiating those 14 unique patterns, it loops over both patterns' weight variable for each of those patterns, creating duplicate patterns based on those weights. As we loop through and create those duplicates, we add the "non related pattern outcome" to each newly instantiated pattern.

After looping through all 14 patterns and their weights, we are left with our trial base of 300 patterns in an array (Study 1, and 200 patterns in an array in Study $2 \& 3$ ). We then shuffle those 300 patterns within the array to ensure no pattern is repeated twice in a row in the array. At this point the data is prepped and ready to be displayed and interacted with.

The next step is to handle those user interactions. The only input we receive from the user is a positive or negative response (they are going steal, they are not going to steal). This input comes from two UI buttons which we have displayed in the GUI.

When a response is received, the application will look at the current pattern being displayed, and run a set of logical operators on that pattern to determine what output we deliver back to the user. We look to see if their answer is the correct answer, as well as if the "non related pattern outcome" aligned with their answer or not. This process is run 300 times, once per trial, and all data is saved in a local set of arrays, ready to be piped into a database configuration of our choosing.

\section{Card Probabilities}

Participants were asked to learn the probabilities of four cards, which were independently associated with each possible outcome (Weather: sun or rain; Crime: steal or no steal) at a fixed probability (see below). 
Supplemental Table 1. Card Pattern Probabilities where 0 represents the absence of a card and 1 represents the presence of a card. Study 1 had a total of 300 trials, whereas study 2 had a total of 200 trials. All the card probabilities remained the same. In order from left to right, card 1, 2, 3, 4.
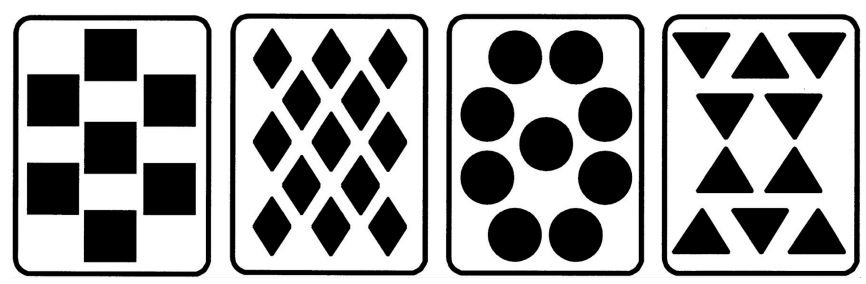

\begin{tabular}{|c|c|c|c|c|c|c|}
\hline Pattern & Card 1 & Card 2 & Card 3 & Card 4 & P (pattern) & P (rain/steal pattern) \\
& & & & & & \\
\hline 1 & 0 & 0 & 0 & 1 & 0.14 & .143 \\
\hline 2 & 0 & 0 & 1 & 0 & 0.08 & .375 \\
\hline 3 & 0 & 0 & 1 & 1 & 0.09 & .111 \\
\hline 4 & 0 & 1 & 0 & 0 & 0.08 & .625 \\
\hline 5 & 0 & 1 & 0 & 1 & 0.06 & .167 \\
\hline 6 & 0 & 1 & 1 & 0 & 0.06 & .5 \\
\hline 7 & 0 & 1 & 1 & 1 & 0.04 & .25 \\
\hline 8 & 1 & 0 & 0 & 0 & 0.14 & .857 \\
\hline 9 & 1 & 0 & 0 & 1 & 0.06 & .5 \\
\hline 10 & 1 & 0 & 1 & 0 & 0.06 & .833 \\
\hline 11 & 1 & 0 & 1 & 1 & 0.03 & .333 \\
\hline 12 & 1 & 1 & 0 & 0 & 0.09 & .889 \\
\hline 13 & 1 & 1 & 0 & 1 & 0.03 & .667 \\
\hline 14 & 1 & 1 & 1 & 0 & 0.04 & .75 \\
\hline
\end{tabular}

\section{Cover Story}

"On each day you are going to see one of eight people/clouds. These [people/clouds] were randomly selected from a database.

Do NOT base your judgment of [steal/rain] or [no steal/sun] on the [person's face/cloud] because it does not have any relevant information about the outcome.

When you make a decision of [steal/rain] or [no steal/sun] always look at the combination of cards! It is the combination of cards that gives high probability information about the outcome."

\section{Comprehension Check Questions}

Participants completed the following questions and were only allowed to proceed once they made a correct choice. 
1. What does the green check mark indicate?

a. Correct Answer: "Good Prediction"

2. What does a combination of cards predict?

a. Correct Answer: "If someone will [insert outcomes based on condition]"

3. Do the Faces/Cloud images have an association with the outcome?

a. Correct Answer: "No"

\section{Face stimuli}

The Face stimuli consisted of one set of two White and two Black male faces in Study 1 (four total) and two new White and Black male Faces in Study 2 \& 3 (eight total) selected from the Chicago Face Database, which were equated across various dimensions.

Supplemental Table 2. Face stimuli used in Study 1. Norm Ratings for faces can be found in CFD 2.0.3 Norming Data and Codebook Excel doc.
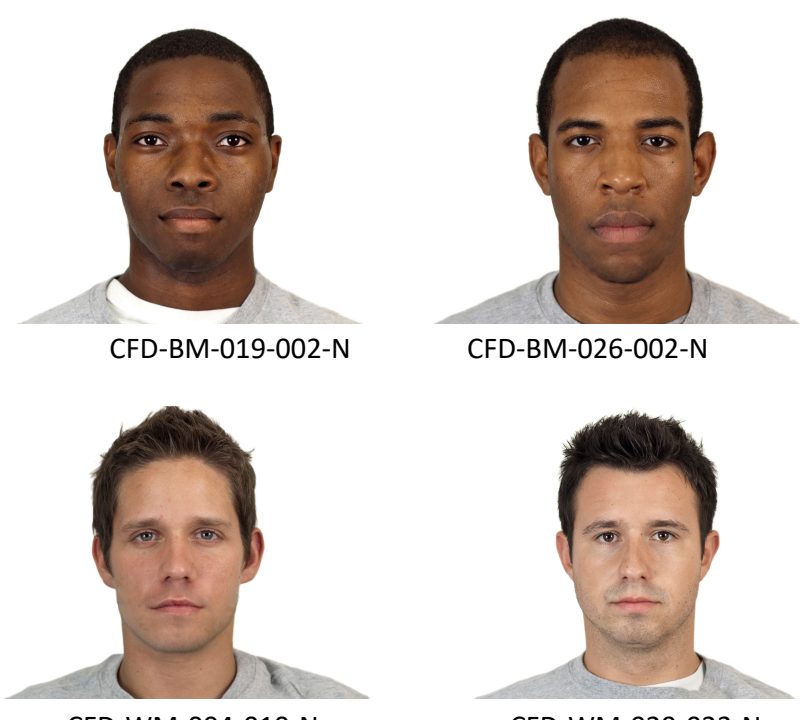

CFD-BM-026-002-N

CFD-WM-004-010-N

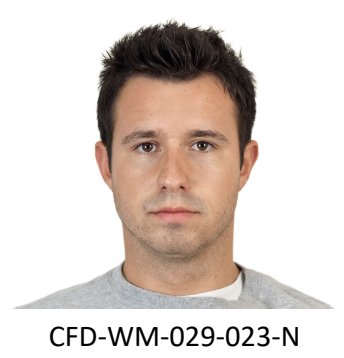


Supplemental Table 3. Face stimuli used in Study 2 and 3. Norm Ratings for faces can be found in CFD 2.0.3 Norming Data and Codebook Excel doc located in the SOM folder.
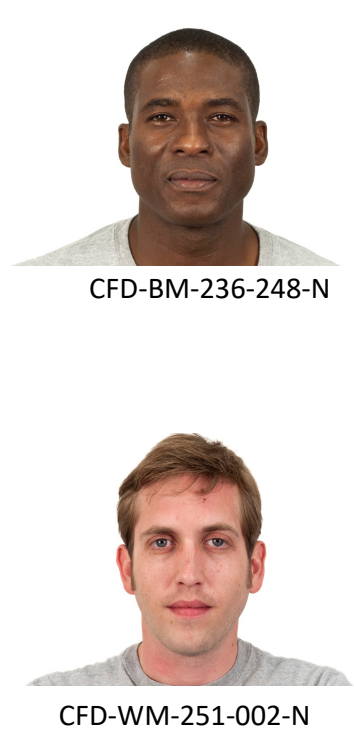

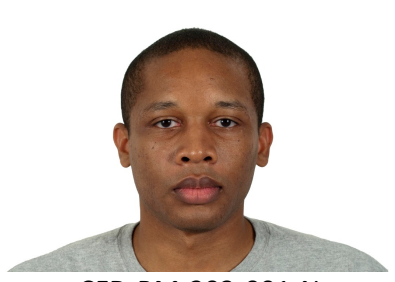

CFD-BM-203-001-N

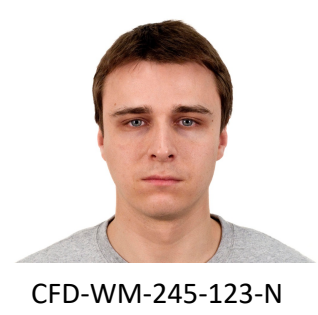

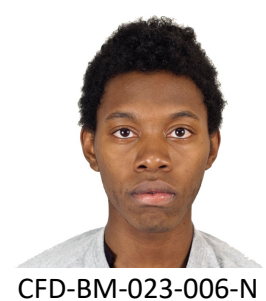

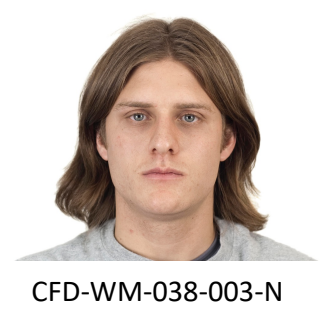

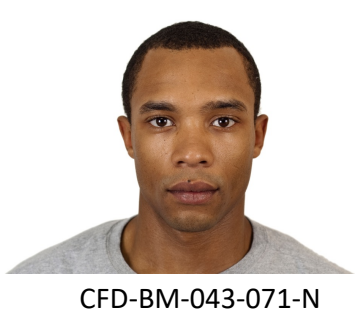

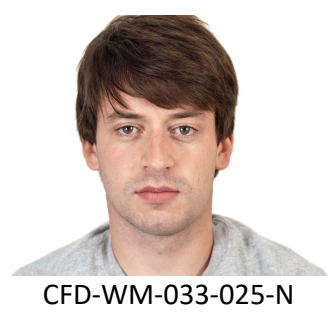

\section{Cloud Stimuli}

Cloud stimuli were piloted with a sample of 22 independent mTurk raters (see Supplemental Table 4 \& 5). 
Supplemental Table 4. Images of clouds that were used in Study 1.
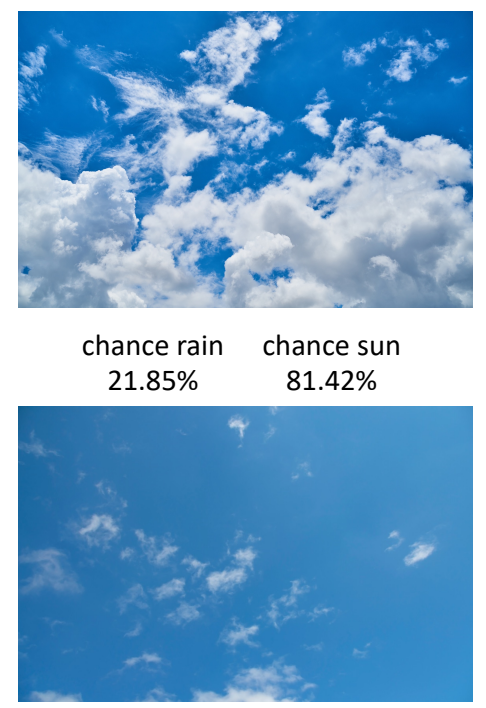

chance rain chance sun $20.8 \%$
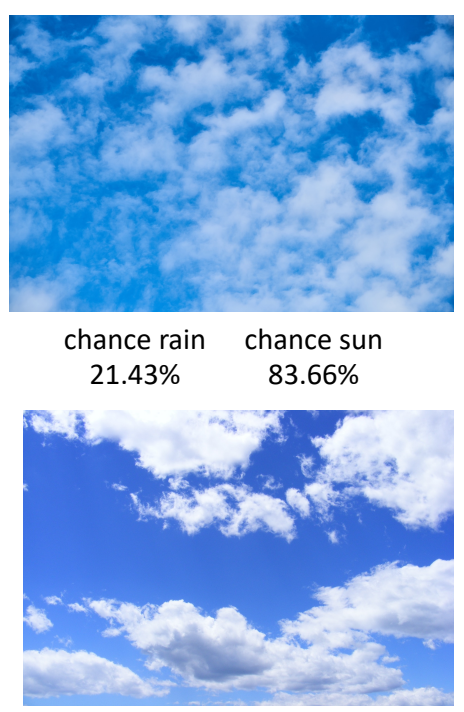

chance rain chance sun $25.23 \% \quad 78.57 \%$

Supplemental Table 5. Images of clouds that were used in Study 2 and 3.

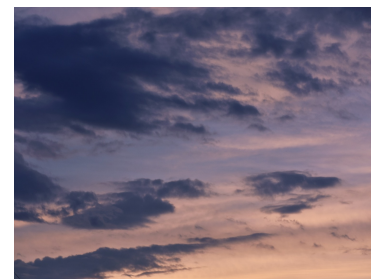

chance rain chance sun $31.25 \%$

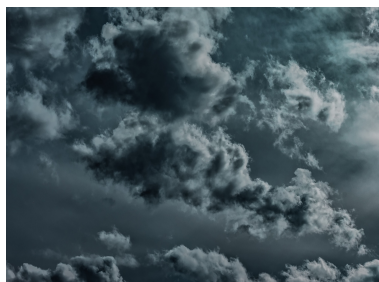

chance rain chance sun $68.27 \%$

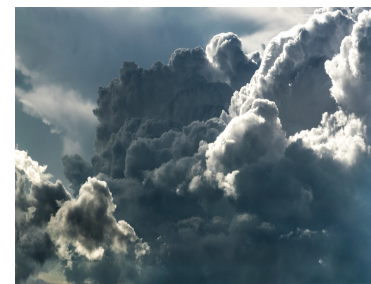

chance rain chance sun $66.91 \%$

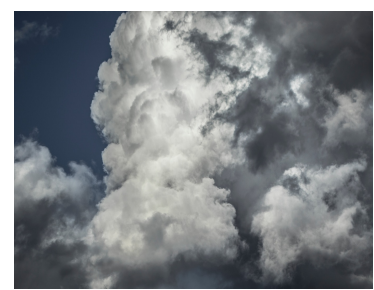

chance rain chance sun $65.26 \% \quad 37.31 \%$

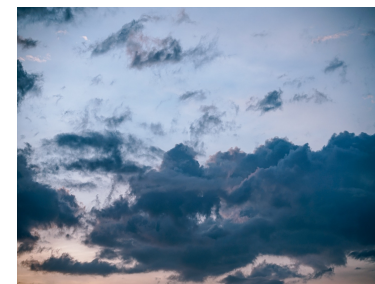

chance rain chance sun $42.59 \% \quad 60.12 \%$

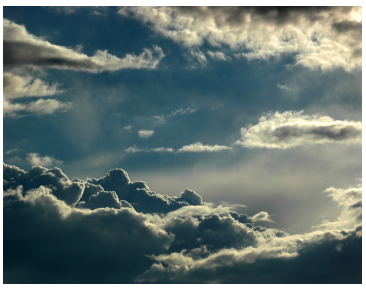

chance rain chance sun $44.17 \% \quad 57.88 \%$

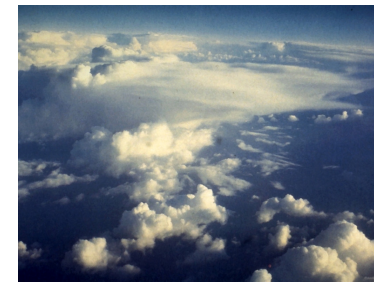

chance rain chance sun $73.45 \% \quad 31.18 \%$

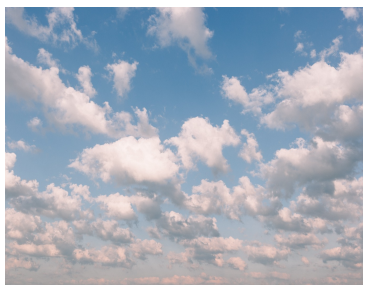

chance rain chance sun $28.72 \% \quad 77.65 \%$ 


\section{Participant Exclusion by condition}

Participants were excluded if they failed to reach an average accuracy of $52 \%$ across trials. Below we report Table 6-8 showing the number of participants excluded in each condition across all studies.

Supplemental Table 6. The number of participants excluded based on condition in Study 1.

\begin{tabular}{|c|c|}
\hline Excluded & N \\
\hline Weather Clouds & 8 \\
\hline Crime Faces & 6 \\
\hline
\end{tabular}

Supplemental Table 7. The number of participants excluded based on condition in Study 2.

\begin{tabular}{|c|c|}
\hline Excluded & N \\
\hline Weather Clouds & 3 \\
\hline Weather Faces & 7 \\
\hline Crime Weather & 3 \\
\hline Crime Faces & 7 \\
\hline
\end{tabular}

Supplemental Table 8. The number of participants excluded based on condition in Study 3 .

\begin{tabular}{|c|c|}
\hline Excluded & $\mathbf{N}$ \\
\hline Athletic & 6 \\
\hline Crime & 8 \\
\hline
\end{tabular}

\section{Individual differences collected but not reported in-text}

We collected a series of different individual differences for exploratory purposes. Below we report each measure that was collected, their reliability (omega), as well as a correlation matrix (Figure 1) showing the Pearson correlation between average accuracy and each measure.

1. Extraversion from the BFI-2 (Soto \& John, 2017; omega $=.89$ )

2. Honesty-Humility facet from HEXACO model of personality (Ashton \& Lee, 2007; Ashton et al., 2014; omega $=.79)$

3. Intergroup Anxiety (Stephan \& Stephan, 1985; omega $=.84$ )

4. General Intergroup Contact and Quantity and Quality scale (Islam \& Hewstone, 1993)

a. Black Contact (omega $=.82$ )

b. White Contact (omega $=.83$ )

c. Black Experience $($ omega $=.71)$

d. White Experience $($ omega $=.82)$

\section{References cited}

Ashton, M. C., \& Lee, K. (2007). Empirical, Theoretical, and Practical Advantages of the HEXACO Model of Personality Structure. Personality and Social Psychology Review, 11(2), 150-166. https://doi.org/10.1177/1088868306294907 
Ashton, M. C., Lee, K., \& de Vries, R. E. (2014). The HEXACO Honesty-Humility, Agreeableness, and Emotionality Factors: A Review of Research and Theory. Personality and Social Psychology Review, 18(2), 139-152. https://doi.org/10.1177/1088868314523838

Islam, M. R., \& Hewstone, M. (1993). Dimensions of Contact as Predictors of Intergroup Anxiety, Perceived Out-Group Variability, and Out-Group Attitude: An Integrative Model. Personality and Social Psychology Bulletin, 19(6), 700-710.

https://doi.org/10.1177/0146167293196005

Stephan, W.G. and Stephan, C.W. (1985), Intergroup Anxiety. Journal of Social Issues, 41: 157175. https://doi.org/10.1111/j.1540-4560.1985.tb01134.x

Soto C.J., John O.P., The next Big Five Inventory (BFI-2): Developing and assessing a hierarchical model with 15 facets to enhance bandwidth, fidelity, and predictive power. $J$ Pers Soc Psychol. 2017 Jul;113(1):117-143. doi: 10.1037/pspp0000096.

Supplemental Figure 1. Pearson correlation matrix between individual difference measures and average accuracy for each condition in Study 2. X-axis represents the strength of the correlation $($ red $=$ positive correlation; blue $=$ negative correlation $)$.

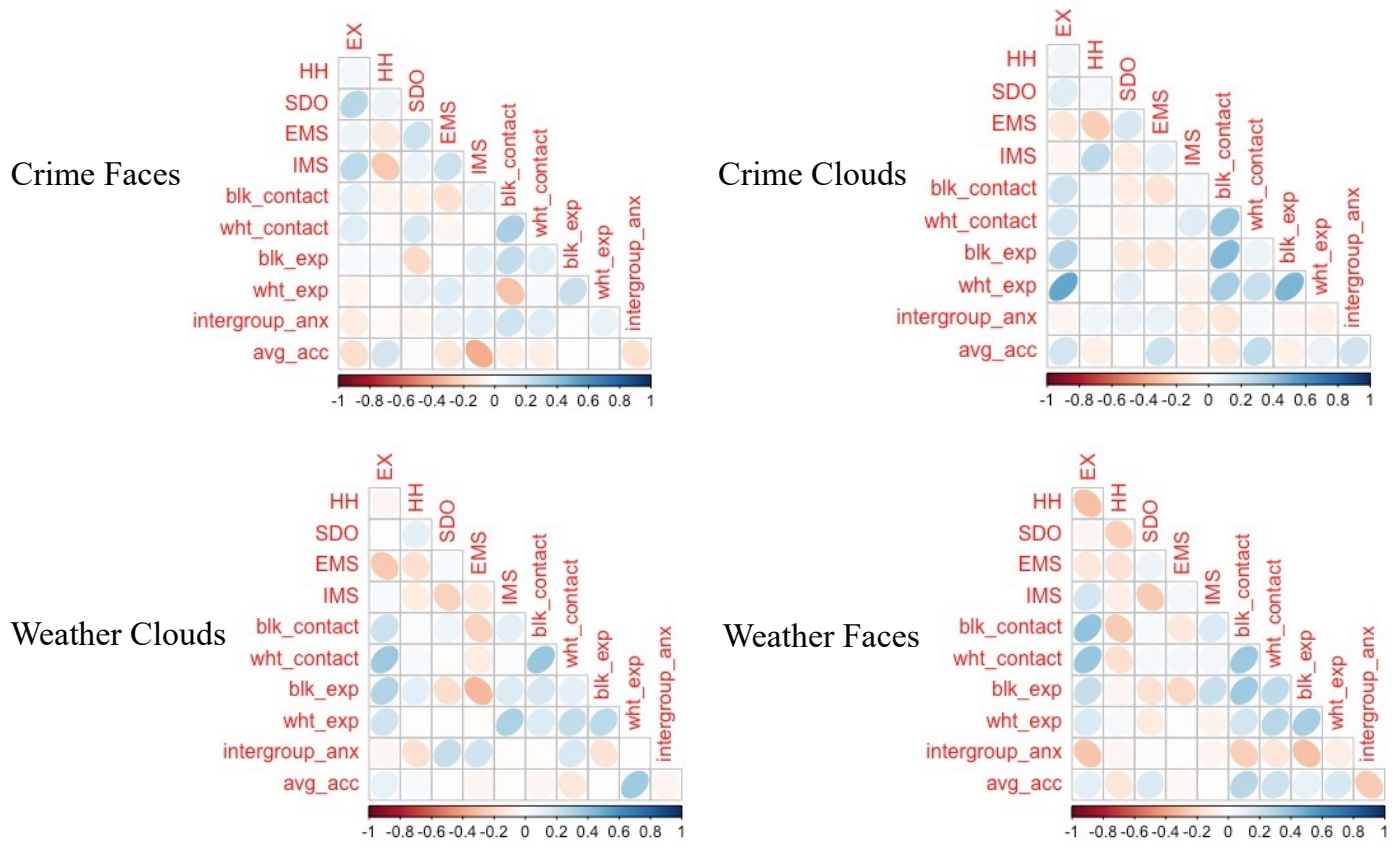

Note: Honesty-Humility (HH), Social Dominance Orientation (SDO), External Motivation to Respond Without Prejudice (EMS), Internal Motivation to Respond Without Prejudice (IMS), Black Contact (blk_contact), White Contact (wht_contact), Black Experience (blk_exp), White Experience (wht_exp), Intergroup Anxiety (intergroup_anx), Average accuracy across trials (avg_acc). 


\section{Supplementary Analyses and Regression Tables}

\section{Changing Exclusion Criterion in Study 1}

To ensure our results were not driven by a $52 \%$ exclusion criterion, we re-ran the analysis with a 50\% exclusion criterion. Importantly, the results in Study 1 do not change with this new threshold.

Supplemental Table 9. Regression table demonstrating that results in Study 1 do not change when the exclusion criterion is changed to $50 \%$.

\begin{tabular}{lccc}
\hline \hline & \multicolumn{3}{c}{ Optimal Response } \\
\multicolumn{1}{c}{ Predictors } & Odds Ratios & $C I$ & $p$ \\
\hline (Intercept) & 3.77 & $3.08-4.62$ & $<\mathbf{0 . 0 0 1}$ \\
Trial & 1.55 & $1.40-1.72$ & $<\mathbf{0 . 0 0 1}$ \\
STIMULI 1 [Weather] & 1.43 & $1.04-1.97$ & $\mathbf{0 . 0 2 9}$ \\
Trial * STIMULI 1 & 1.11 & $0.94-1.30$ & 0.218 \\
[Weather] & & &
\end{tabular}

\section{Random Effects}

$\begin{array}{ll}\sigma^{2} & 3.29 \\ \tau_{00 \text { P. }} & 0.63 \\ \tau_{11 \text { P..scale(Trial) }} & 0.14 \\ \rho_{01 \text { P. }} & 0.69 \\ \text { ICC } & 0.19 \\ \mathrm{~N}_{\text {P. }} & 103\end{array}$

Observations 30497

Marginal $\mathrm{R}^{2} /$ Conditional $\mathrm{R}^{2} \quad 0.060$ / 0.239

\section{Participant race as a moderator in Study 2}

To test whether participant race moderated learning, we tested the interaction of selfidentified race by condition. Given that our sample contained a relatively small proportion of self-identified "White", "Black" and "Other" participants, we report one model where each race category is coded as a separate factor (effects coded with "Other" coded as -1; see Table 10), as 
STEREOTYPES DISRUPT LEARNING

well as a second model where "White", "Black" and "Other" are collapsed into a single "Other" factor (see Supplemental Table 11).

Supplemental Table 10. Mixed model examining the interaction between condition and selfidentified race in Study 2. Each race category was modeled as a separate factor.

\begin{tabular}{|c|c|c|c|}
\hline \multirow[b]{2}{*}{ Predictors } & \multicolumn{3}{|c|}{ acc } \\
\hline & Odds Ratios & $C I$ & $p$ \\
\hline (Intercept) & 5.93 & $4.98-7.06$ & $<0.001$ \\
\hline Trial & 1.74 & $1.67-1.82$ & $<0.001$ \\
\hline Condition dum [steal] & 0.74 & $0.58-0.95$ & 0.018 \\
\hline $\begin{array}{l}\text { Condition dum [steal } \\
\text { clouds] }\end{array}$ & 1.03 & $0.79-1.34$ & 0.822 \\
\hline $\begin{array}{l}\text { Condition dum [weather } \\
\text { faces] }\end{array}$ & 0.81 & $0.64-1.04$ & 0.104 \\
\hline ethnicity effBlack & 0.92 & $0.53-1.61$ & 0.780 \\
\hline ethnicity eff [Latinx] & 0.84 & $0.66-1.08$ & 0.184 \\
\hline $\begin{array}{l}\text { ethnicity eff [Native } \\
\text { Hawaiian or Pacific } \\
\text { Islander] }\end{array}$ & 0.83 & $0.25-2.73$ & 0.758 \\
\hline ethnicity eff [Other] & 0.96 & $0.61-1.51$ & 0.857 \\
\hline ethnicity eff [White] & 1.24 & $0.82-1.87$ & 0.319 \\
\hline $\begin{array}{l}\text { Condition dum [steal] } \\
\text { ethnicity effBlack }\end{array}$ & 1.17 & $0.61-2.23$ & 0.637 \\
\hline $\begin{array}{l}\text { Condition dum [steal } \\
\text { clouds] * ethnicity } \\
\text { effBlack }\end{array}$ & 1.74 & $0.78-3.89$ & 0.180 \\
\hline $\begin{array}{l}\text { Condition dum [weather } \\
\text { faces] } * \text { ethnicity } \\
\text { effBlack }\end{array}$ & 1.08 & $0.49-2.36$ & 0.849 \\
\hline
\end{tabular}




\begin{tabular}{|c|c|c|c|}
\hline $\begin{array}{l}\text { Condition dum [steal] } \\
\text { ethnicity eff [Latinx] }\end{array}$ & 1.12 & $0.77-1.63$ & 0.563 \\
\hline $\begin{array}{c}\text { Condition dum [steal } \\
\text { clouds] * ethnicity eff } \\
\text { [Latinx] }\end{array}$ & 0.93 & $0.64-1.37$ & 0.718 \\
\hline $\begin{array}{l}\text { Condition dum [weather } \\
\text { faces] }{ }^{*} \text { ethnicity eff } \\
\text { [Latinx] }\end{array}$ & 1.02 & $0.69-1.51$ & 0.923 \\
\hline $\begin{array}{c}\text { Condition dum [steal] } * \\
\text { ethnicity eff [Native } \\
\text { Hawaiian or Pacific } \\
\text { Islander] }\end{array}$ & 0.57 & $0.11-3.03$ & 0.513 \\
\hline $\begin{array}{l}\text { Condition dum [weather } \\
\text { faces] } * \text { ethnicity eff } \\
\text { [Native Hawaiian or } \\
\text { Pacific Islander] }\end{array}$ & 1.37 & $0.31-6.04$ & 0.679 \\
\hline $\begin{array}{l}\text { Condition dum [steal] } \\
\text { ethnicity eff [Other] }\end{array}$ & 1.02 & $0.50-2.08$ & 0.965 \\
\hline $\begin{array}{c}\text { Condition dum [steal } \\
\text { clouds] } * \text { ethnicity eff } \\
\text { [Other] }\end{array}$ & 0.78 & $0.39-1.56$ & 0.484 \\
\hline $\begin{array}{c}\text { Condition dum [weather } \\
\text { faces] }{ }^{*} \text { ethnicity eff } \\
\text { [Other] }\end{array}$ & 1.12 & $0.59-2.13$ & 0.721 \\
\hline $\begin{array}{l}\text { Condition dum [steal] * } \\
\text { ethnicity eff [White] }\end{array}$ & 0.75 & $0.42-1.35$ & 0.333 \\
\hline $\begin{array}{c}\text { Condition dum [steal } \\
\text { clouds] } * \text { ethnicity eff } \\
\text { [White] }\end{array}$ & 0.65 & $0.35-1.21$ & 0.178 \\
\hline $\begin{array}{l}\text { Condition dum [weather } \\
\text { faces] }{ }^{*} \text { ethnicity eff } \\
\text { [White] }\end{array}$ & 1.15 & $0.66-2.02$ & 0.620 \\
\hline \multicolumn{4}{|c|}{ Random Effects } \\
\hline$\sigma^{2}$ & & 3.29 & \\
\hline$\tau_{00 \text { Participant }}$ & & 0.46 & \\
\hline
\end{tabular}


STEREOTYPES DISRUPT LEARNING

$\begin{array}{cc}\tau_{00 \text { Face_Shown }} & 0.00 \\ \tau_{11 \text { Participant.scale(Trial) }} & 0.13 \\ \rho_{01 \text { Participant }} & 0.59 \\ \text { ICC } & 0.15 \\ \mathrm{~N}_{\text {Participant }} & 363 \\ \mathrm{~N}_{\text {Face_Shown }} & 8 \\ \text { Observations } & 72008 \\ \text { Marginal } \mathrm{R}^{2} / \text { Conditional } \mathrm{R}^{2} & 0.081 / 0.220\end{array}$

Supplemental Table 11. Mixed model examining the interaction between condition and selfidentified race in Study 2. This model collapses the three race categories with the fewest participants ("White", "Black," and "Other") into a single factor ("Other:").

\begin{tabular}{cccc}
\hline \hline & \multicolumn{3}{c}{ acc } \\
Predictors & Odds Ratios & $C I$ & $p$ \\
\hline (Intercept) & 6.23 & $4.86-7.99$ & $<\mathbf{0 . 0 0 1}$ \\
Trial & 1.74 & $1.67-1.82$ & $<\mathbf{0 . 0 0 1}$ \\
Condition dum [steal] & 0.68 & $0.49-0.95$ & $\mathbf{0 . 0 2 5}$ \\
$\begin{array}{c}\text { Condition dum [steal } \\
\text { clouds] }\end{array}$ & 0.92 & $0.63-1.33$ & 0.650 \\
$\begin{array}{c}\text { Condition dum [weather } \\
\text { faces] }\end{array}$ & 0.94 & $0.67-1.32$ & 0.728 \\
$\begin{array}{c}\text { ethnicity recode [Latinx] } \\
\text { ethnicity recode [Asian] }\end{array}$ & 0.80 & $0.58-1.11$ & 0.185 \\
$\begin{array}{c}\text { Condition dum [steal] * } \\
\text { ethnicity recode [Latinx] }\end{array}$ & 1.22 & $0.77-1.92$ & 0.402 \\
$\begin{array}{c}\text { Condition dum [steal } \\
\text { clouds] * ethnicity } \\
\text { recode [Latinx] }\end{array}$ & 1.05 & $0.65-1.68$ & 0.850 \\
\hline
\end{tabular}


STEREOTYPES DISRUPT LEARNING

$\begin{array}{llll}\begin{array}{l}\text { Condition dum [weather } \\ \text { faces] * ethnicity recode } \\ \quad \text { [Latinx] }\end{array} & 0.88 & 0.55-1.41 & 0.596 \\ \begin{array}{l}\text { Condition dum [steal] * } \\ \text { ethnicity recode [Asian] }\end{array} & 1.06 & 0.72-1.57 & 0.776 \\ \begin{array}{c}\text { Condition dum [steal } \\ \text { clouds] * ethnicity } \\ \text { recode [Asian] }\end{array} & 1.12 & 0.71-1.77 & 0.623 \\ \begin{array}{c}\text { Condition dum [weather } \\ \text { faces] * ethnicity recode }\end{array} & 0.86 & 0.57-1.31 & 0.488 \\ \quad \text { [Asian] }\end{array}$

\begin{tabular}{cc}
\multicolumn{2}{c}{ Random Effects } \\
$\sigma^{2}$ & 3.29 \\
$\tau_{00 \text { Participant }}$ & 0.46 \\
$\tau_{00 \text { Face_Shown }}$ & 0.00 \\
$\tau_{11}$ Participant.scale(Trial) & 0.13 \\
$\rho_{01 \text { Participant }}$ & 0.57 \\
ICC & 0.15 \\
N Participant & 363 \\
$\mathrm{~N}_{\text {Face_Shown }}$ & 8 \\
Observations & 72008 \\
Marginal R ${ }^{2} /$ Conditional $\mathrm{R}^{2}$ & $0.079 / 0.219$
\end{tabular}

\section{Participant race as a moderator in Study 3}

To test whether participant race moderated learning, we tested the interaction of selfidentified race by condition

Supplemental Table 12. Mixed model examining the interaction between condition and self-identified race in Study 3.

\begin{tabular}{lccc}
\hline \hline & \multicolumn{4}{c}{ acc } \\
Predictors & Odds Ratios & $C I$ & $p$ \\
\hline (Intercept) & 4.97 & $4.08-6.06$ & $<\mathbf{0 . 0 0 1}$
\end{tabular}


STEREOTYPES DISRUPT LEARNING

\begin{tabular}{|c|c|c|c|}
\hline Trial & 1.78 & $1.69-1.87$ & $<0.001$ \\
\hline Condition dumTouchdown & 0.93 & $0.69-1.26$ & 0.655 \\
\hline ethnicity eff2 & 0.84 & $0.42-1.69$ & 0.633 \\
\hline ethnicity eff3 & 0.81 & $0.61-1.07$ & 0.140 \\
\hline ethnicity eff4 & 0.96 & $0.54-1.71$ & 0.896 \\
\hline ethnicity eff5 & 1.31 & $0.85-2.02$ & 0.219 \\
\hline $\begin{array}{l}\text { Condition dumTouchdown * } \\
\text { ethnicity eff } 2\end{array}$ & 0.57 & $0.13-2.57$ & 0.465 \\
\hline $\begin{array}{l}\text { Condition dumTouchdown * } \\
\text { ethnicity eff3 }\end{array}$ & 1.32 & $0.86-2.02$ & 0.209 \\
\hline $\begin{array}{l}\text { Condition dumTouchdown * } \\
\text { ethnicity eff } 4\end{array}$ & 1.08 & $0.47-2.46$ & 0.859 \\
\hline $\begin{array}{l}\text { Condition dumTouchdown * } \\
\text { ethnicity eff5 }\end{array}$ & 0.60 & $0.29-1.25$ & 0.170 \\
\hline \multicolumn{4}{|l|}{ Random Effects } \\
\hline$\sigma^{2}$ & 3.29 & & \\
\hline$\tau_{00 \text { Participant }}$ & 0.52 & & \\
\hline$\tau_{00 \text { Face_Shown }}$ & 0.00 & & \\
\hline$\tau_{11 \text { Participant.scale(Trial) }}$ & 0.08 & & \\
\hline$\rho_{01 \text { Participant }}$ & 0.47 & & \\
\hline $\mathrm{ICC}$ & 0.15 & & \\
\hline $\mathrm{N}_{\text {Participant }}$ & 176 & & \\
\hline $\mathrm{N}$ Face_Shown & 8 & & \\
\hline Observations & 34807 & & \\
\hline Marginal $\mathrm{R}^{2}$ / Conditional $\mathrm{R}^{2}$ & 0.083 & & \\
\hline
\end{tabular}

\section{Effect size comparison in Study 2}


STEREOTYPES DISRUPT LEARNING

To quantify the difference in learning between the Weather Face and Crime Face condition, we calculated the percent accuracy change. To do so, consider the Crime Face condition as the base rate for learning performance (4286 [incorrect] / (14028 [correct] +4286 [incorrect $]=0.2340286$ ). We can use the odds ratio representing the difference in learning between the Crime Face and Weather Face to calculate the percent accuracy change between these two conditions (see Supplemental Figure 2). Doing so reveals an incorrect rate of 14.69\% in the Weather Face relative to $23.40 \%$ in the Crime Face condition. In practical terms, the error rate increases by over $50 \%$ when participants learn in an environment where faces are stereotypically associated with predicted outcomes relative to when they are not.

Formula for calculating the percent accuracy change between the Crime Face and Weather Face condition in Study 2.

$$
\frac{O R(\text { WeatherFace }) * p(\text { FaceCrime })}{1+\text { OR }(\text { WeatherFace }) * p(\text { FaceCrime })-p(\text { FaceCrime })}=\frac{(.814 * .234)}{1+(.814 * .234)-.234}=.1469
$$

\section{Bayesian Analysis in Study 3}

We tested likelihood that the observed null effect of condition was true with a multilevel Bayesian logistic model using the brms package in $\mathrm{R}$. This method provides a more appropriate test for the probability of obtaining a null effect based on the proportion of the posterior distribution of the parameter estimate that falls within a range that would be considered negligible (i.e., the region of practical equivalence).

Supplemental Figure 2. Credibility intervals generated from the Bayesian model in Study 3. 


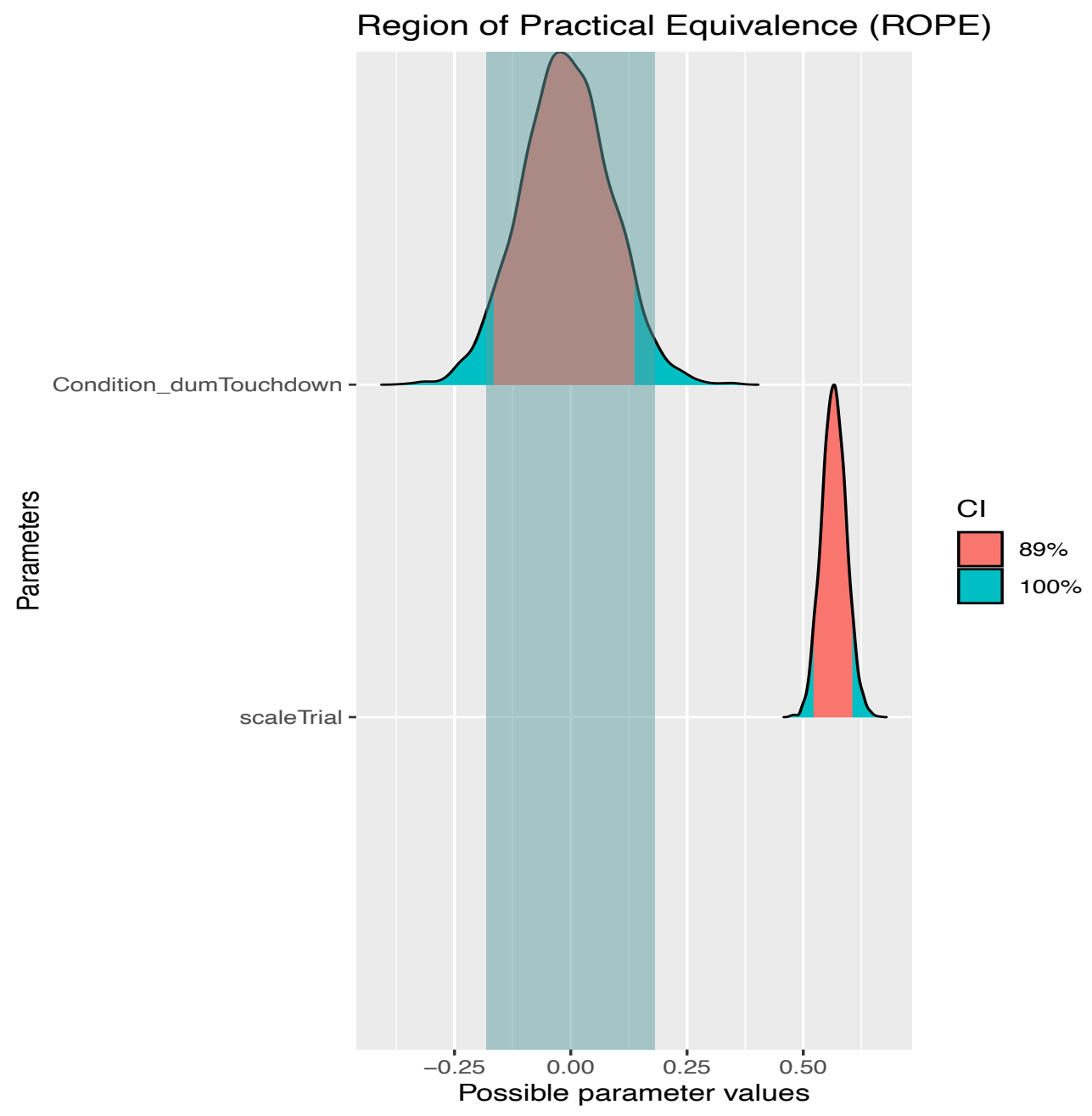

\section{First-order effects Aggregated Analysis}

We estimated a series of models to test whether learning was affected at the trial-level. The trial variable was first estimated as a covariate, and then subsequently as a moderator. All models examining behavior in the Crime condition included a dummy coded covariate to control for study. Each model was tested both in the Crime Face (see Supplemental Table 13) and Athletic condition (see Supplemental Table 14). 
STEREOTYPES DISRUPT LEARNING

Supplemental Table 13. First order effects in Crime Face condition reported in Aggregated Analysis.

\begin{tabular}{|c|c|c|c|c|}
\hline & Fixed effects & $b(S E)$ & $z / t$ & $p$ \\
\hline \multirow{4}{*}{ Model 1} & Intercept & $1.51(.08)$ & $19.16(z)$ & $p<.0001$ \\
\hline & Trial & $.53(.03)$ & $17.16(z)$ & $p<.0001$ \\
\hline & stimuli (white) & $-.002(.03)$ & $-.071(z)$ & $p=.94$ \\
\hline & Trial $x$ stimuli & $-.07(.10)$ & $-.76(z)$ & $p=.44$ \\
\hline \multirow{4}{*}{ Model 2} & Intercept & $1.49(.07)$ & $19.16(z)$ & $p<.0001$ \\
\hline & Trial & $.52(.03)$ & $17.16(z)$ & $p<.0001$ \\
\hline & Stereotype congruent & $.037(.02)$ & $1.137(z)$ & $p=.16$ \\
\hline & Trial x Stereotype congruent & $.003(.02)$ & $.132(z)$ & $p=.89$ \\
\hline \multirow{4}{*}{ Model 3} & Intercept & $.47(.02)$ & $22.73(t)$ & $p<.0001$ \\
\hline & Trial & $-.067(.007)$ & $-8.89(t)$ & $p<.0001$ \\
\hline & Stereotype congruent & $.003(.004)$ & $.064(t)$ & $p=.94$ \\
\hline & Trial x Stereotype congruent & $-.005(.004)$ & $-.118(t)$ & $p=.906$ \\
\hline \multirow{3}{*}{ Model 4} & Intercept & $-.059(.02)$ & $-2.92(z)$ & $p=.003$ \\
\hline & Trial & $-.01(.011)$ & $-1.42(z)$ & $p=.159$ \\
\hline & Predictive weight (categorical) & NA & NA & $p$-values $>.11$ \\
\hline \multirow{4}{*}{ Model 5} & Intercept & $-.045(.018)$ & $-2.42(z)$ & $p=.015$ \\
\hline & Trial & $-.016(.011)$ & $-1.44(z)$ & $p=.49$ \\
\hline & Predictive weight (absolute) & $.013(.01)$ & $1.27(z)$ & $p=.20$ \\
\hline & $\begin{array}{c}\text { Trial x Predictive weight } \\
\text { (absolute) }\end{array}$ & $.017(.01)$ & $.67(z)$ & $p=.50$ \\
\hline \multirow{4}{*}{ Model 6} & Intercept & $-.02(.01)$ & $1.37(z)$ & $p=.18$ \\
\hline & Trial & $-.02(.01)$ & $-1.99(z)$ & $p=.046$ \\
\hline & SDO & $-.011(.01)$ & $-1.019(z)$ & $p=.30$ \\
\hline & Trial $x$ SDO & $-.007(.01)$ & $-.67(z)$ & $p=.49$ \\
\hline \multirow{4}{*}{ Model 7} & Intercept & $.016(.016)$ & $.998(z)$ & $p=.318$ \\
\hline & Trial & $-.018(.011)$ & $-1.613(z)$ & $p=.107$ \\
\hline & IMS & $-.006(.011)$ & $-.559(z)$ & $p=.549$ \\
\hline & Trial $\times$ IMS & $.017(.011)$ & $1.499(z)$ & $p=.134$ \\
\hline
\end{tabular}


STEREOTYPES DISRUPT LEARNING

Supplemental Table 14. First order effects for Athletic condition reported in Aggregated Analysis.

\begin{tabular}{|c|c|c|c|c|}
\hline & Fixed effects & $b(S E)$ & $z / t$ & $p$ \\
\hline \multirow{4}{*}{ Model 1} & Intercept & $1.57(.08)$ & $18.96(z)$ & $p<.0001$ \\
\hline & Trial & $.61(.04)$ & $14.98(z)$ & $p<.0001$ \\
\hline & stimuli (white) & $.01(.04)$ & $.33(z)$ & $p=.73$ \\
\hline & Trial x stimuli & $-.007(.04)$ & $-.71(z)$ & $p=.86$ \\
\hline \multirow{4}{*}{ Model 2} & Intercept & $1.57(.07)$ & $19.78(z)$ & $p<.0001$ \\
\hline & Trial & $.58(.04)$ & $14.36(z)$ & $p<.0001$ \\
\hline & stimuli (white) & $.02(.04)$ & $.51(z)$ & $p=.607$ \\
\hline & Trial x stimuli & $.05(.04)$ & $1.31(z)$ & $p=.18$ \\
\hline \multirow{4}{*}{ Model 3} & Intercept & $.047(.02)$ & $23.67(t)$ & $p<.0001$ \\
\hline & Trial & $-.06(.009)$ & $-6.37(t)$ & $p<.0001$ \\
\hline & Stereotype congruent & $-.001(.006)$ & $-.245(t)$ & $p=.80$ \\
\hline & Trial $x$ Stereotype congruent & $.005(.006)$ & $.81(t)$ & $p=.41$ \\
\hline \multirow{3}{*}{ Model 4} & Intercept & $.006(.02)$ & $.31(z)$ & $p=.75$ \\
\hline & Trial & $-.04(.01)$ & $-2.71(z)$ & $p=.006$ \\
\hline & Predictive weight (categorical) & NA & NA & $p$-values $>.25$ \\
\hline \multirow{4}{*}{ Model 5} & Intercept & $-.001(.01)$ & $-.08(z)$ & $p=.93$ \\
\hline & Trial & $-.04(.01)$ & $-2.707(z)$ & $p=.006$ \\
\hline & Predictive weight (absolute) & $-.01(.01)$ & $-.709(z)$ & $p=.47$ \\
\hline & Trial $x$ Predictive weight (absolute) & $.002(.01)$ & $.18(z)$ & $p=.85$ \\
\hline \multirow{4}{*}{ Model 6} & Intercept & $-.009(.01)$ & $-.51(z)$ & $p=.60$ \\
\hline & Trial & $-.04(.01)$ & $-2.59(z)$ & $p=.009$ \\
\hline & SDO & $-.02(.01)$ & $1.47(z)$ & $p=.14$ \\
\hline & Trial $x$ SDO & $-.009(.01)$ & $-.54(z)$ & $p=.58$ \\
\hline \multirow{4}{*}{ Model 7} & Intercept & $-.01(.01)$ & $-.75(z)$ & $p=.44$ \\
\hline & Trial & $-.04(.01)$ & $-2.503(z)$ & $p=.01$ \\
\hline & IMS & $.01(.01)$ & $.78(z)$ & $p=.43$ \\
\hline & Trial $x$ IMS & $.003(.01)$ & $.17(z)$ & $p=.86$ \\
\hline
\end{tabular}

\section{Second-order effects Aggregated Analysis}

In addition, we estimated a series of analyses that tested whether learning was instead disrupted due second-order effects that accumulate across the task. Each model was tested both in the Crime Face (see Supplemental Table 15) and Athletic condition (see Supplemental Table 16). 
STEREOTYPES DISRUPT LEARNING

Supplemental Table 15. Second order effects for Crime Face condition reported in Aggregated Analysis.

\begin{tabular}{|c|c|c|c|c|}
\hline \multirow{5}{*}{ Model 1} & Fixed effects & $b(S E)$ & $t / z$ & $p$ \\
\hline & Intercept & $1.518(.077)$ & $19.614(z)$ & $p<.0001$ \\
\hline & Trial & $.54(.027)$ & $19.49(z)$ & $p<.0001$ \\
\hline & IMS & $-.129(.05)$ & $-2.24(z)$ & $p=.024$ \\
\hline & Trial x IMS & $-.06(.027)$ & $-2.405(z)$ & $p=.016$ \\
\hline \multirow{4}{*}{ Model 2} & Intercept & $1.507(.07)$ & $19.55(z)$ & $p<.0001$ \\
\hline & Trial & $.53(.027)$ & $19.42(z)$ & $p<.0001$ \\
\hline & EMS & $-.03(.04)$ & $-.98(z)$ & $p=.425$ \\
\hline & Trial $\times$ EMS & $-.04(.026)$ & $-1.87(z)$ & $p=.061$ \\
\hline \multirow{6}{*}{ Model 3} & Intercept & $1.51(.07)$ & $19.552(z)$ & $p<.0001$ \\
\hline & Trial & $.54(.02)$ & $19.612(z)$ & $p<.0001$ \\
\hline & IMS & $-.12(.06)$ & $-2.14(z)$ & $p=.032$ \\
\hline & EMS & $-.04(.05)$ & $-.82(z)$ & $p=.40$ \\
\hline & IMS $\times$ EMS & $-.03(.05)$ & $-.62(z)$ & $p=.53$ \\
\hline & Trial $\times$ IMS $\times$ EMS & $-.009(.02)$ & $-.41(z)$ & $p=.68$ \\
\hline \multirow{5}{*}{ Model 4} & Intercept & $\begin{array}{l}.48(.021) \\
\end{array}$ & $22.71(t)$ & $p<.0001$ \\
\hline & Trial & $-.069(.007)$ & $-9.44(t)$ & $p<.0001$ \\
\hline & IMS & $.015(.014)$ & $1.031(t)$ & $p=.30$ \\
\hline & EMS & $.001(.009)$ & $.12(t)$ & $p=.90$ \\
\hline & Trial $x$ IMS & $.02(.007)$ & $2.88(t)$ & $p=.0046$ \\
\hline \multirow{4}{*}{ Model 5} & Intercept & $.48(.02)$ & $23.06(t)$ & $p<.0001$ \\
\hline & Trial & $-.068(.007)$ & $-9.412(t)$ & $p<.0001$ \\
\hline & Stimuli [White Faces] & $-.009(.004)$ & $-1.998(t)$ & $p=.045$ \\
\hline & Trial $\times$ Stimuli [White Faces] & $.04(.029)$ & $1.53(t)$ & $p=.12$ \\
\hline
\end{tabular}

Supplemental Table 16. Second order effects for Athletic condition reported in Aggregated Analysis.

\begin{tabular}{|c|c|c|c|c|}
\hline & Fixed effects & $b(S E)$ & $t / z$ & $p$ \\
\hline \multirow[b]{3}{*}{ M1 } & Intercept & $1.56(.08)$ & $18.709(z)$ & $p<.0001$ \\
\hline & Trial & $.61(.03)$ & $15.39(z)$ & $p<.0001$ \\
\hline & IMS & $.03(.08)$ & $.43(z)$ & $p=.662$ \\
\hline \multirow{4}{*}{ M2 } & Trial $x$ IMS & $-.003(.03)$ & $-.077(z)$ & $p=.93$ \\
\hline & Intercept & $1.55(.083)$ & $18.57(z)$ & $p<.0001$ \\
\hline & Trial & $.61(.039)$ & $15.67(z)$ & $p<.0001$ \\
\hline & EMS & $.03(.08)$ & $.43(z)$ & $p=.665$ \\
\hline \multirow{6}{*}{ M3 } & Trial $x$ EMS & $-02(.03)$ & $.52(z)$ & $p=.59$ \\
\hline & Intercept & $1.56(.08)$ & $18.24(z)$ & $p<.0001$ \\
\hline & Trial & $.61(.03)$ & $15.62(z)$ & $p<.0001$ \\
\hline & IMS & $.04(.09)$ & $.42(z)$ & $p=.67$ \\
\hline & EMS & $.03(.08)$ & $.45(z)$ & $p=.65$ \\
\hline & IMS x EMS & $.016(.08)$ & $.199(z)$ & $p=.82$ \\
\hline \multirow{8}{*}{ M4 } & Trial $\mathrm{x}$ IMS $\mathrm{x}$ EMS & $-.039(.03)$ & $-1.01(z)$ & $p=.31$ \\
\hline & Intercept & $.48(.02)$ & $23.43(t)$ & $p<.0001$ \\
\hline & Trial & $-.06(.009)$ & $-6.56(t)$ & $p<.0001$ \\
\hline & IMS & $.02(.021)$ & $1.022(t)$ & $p=.31$ \\
\hline & EMS & .001 (.02) & $.094(t)$ & $p=.92$ \\
\hline & Trial x IMS & $.003(.009)$ & $.34(t)$ & $p=.73$ \\
\hline & Intercept & $.47(.02)$ & $23.72(t)$ & $p<.0001$ \\
\hline & Trial & $-.058(.009)$ & $-5.99(t)$ & $p<.0001$ \\
\hline \multirow{2}{*}{ M5 } & Stimuli [White Faces] & $-.01(.006)$ & $-1.59(t)$ & $p=.11$ \\
\hline & Trial $\times$ Stimuli [White Faces] & $-.001(.006)$ & $-.22(t)$ & $p=.82$ \\
\hline
\end{tabular}




\section{Association between IMS and accuracy in the presence of faces}

To examine whether IMS impact learning in the presence of Black and White faces, even in the absence of stereotypical associations, we also tested whether learning was influenced by IMS in the Weather Face condition and whether this effect differed from the Crime Face condition. To do so, we estimated a model which included the Weather Face condition (in addition to the Crime Face [reference]). This model demonstrated a significant IMS by condition interaction $(\beta=.197,95 \% C I S=[-.24,-.01], S E=.09, z=2.187, O R=1.22, p=.028$; see Supplemental Figure 4). This demonstrates that higher IMS leads to worse accuracy in the Crime Face but not Weather Face condition, suggesting that it is learning in the presence of stereotypes, rather than learning in the presence of Black and White faces alone, that disrupts learning for individuals higher in IMS.

Supplemental Figure 4. examining the predicted effects for IMS by condition interaction demonstrating that IMS only leads to worse performance in the Crime Face but not Weather

Face.

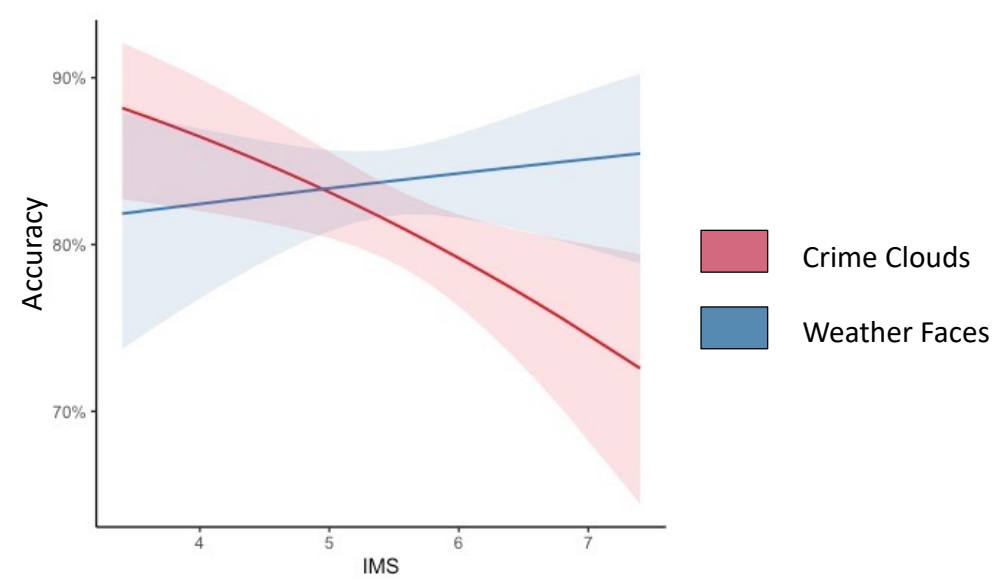

\section{Reinforcement Learning Models in Study 2}

We pre-registered a series of reinforcement learning models to investigate the underlying mechanisms of learning in social and nonsocial contexts. Reinforcement learning characterizes learning in terms of maximizing reward (e.g., correct responses) and minimizing punishment (e.g., incorrect responses; see Daw et al., 2006). RL thus enables us to test competing hypotheses about the mechanisms underlying learning decrements by constraining learning rates to vary either as a function of trial-level features (e.g., stimuli) or as a function of cumulative temporal components (e.g., failure to incorporate feedback), using model comparison to determine the best fitting model. More specifically, these models allow us to determine whether learning is disrupted due to stereotype-congruent applications on a trial-by-trial basis, or by cumulative effect over time due to stereotype inhibition.

\section{Method}

Our models were based off of the Rescorla-Wagner reinforcement learning model (Rescorla \& Wagner, 1972). In this model, the learning rate $(\alpha)$ controls the extent to which 
expectation violations (i.e. prediction errors) are incorporated and contribute to the updating of expectations and behaviors. The model appears as follows:

$\mathrm{W}_{\mathrm{t}+1}\left(\mathrm{G}_{\mathrm{t}}\right)=\mathrm{W}_{\mathrm{t}}\left(\mathrm{G}_{\mathrm{t}}\right)+\alpha * \delta_{\mathrm{t}}$

The model describes how the expected outcome $\left(\mathrm{W}_{\mathrm{t}+1}\right)$ given a card or card pattern $\left(\mathrm{G}_{\mathrm{t}}\right)$ is updated from its existing value $\left(\mathrm{W}_{\mathrm{t}}\right)$ according to the prediction error term $\left(\delta_{t}\right)$ and how much of the error is incorporated by the learning rate. The error term is computed as:

$$
\delta_{\mathrm{t}}=\mathrm{O}_{\mathrm{t}}-\mathrm{W}_{\mathrm{t}}\left(\mathrm{G}_{\mathrm{t}}\right)+\varepsilon_{\mathrm{t}}
$$

Therefore, the error term is the difference between the observed outcome $\left(\mathrm{O}_{\mathrm{t}}\right)$ and the expectation given the present set of cards. Each card was set to an initial associative weight of 0 at the start of the trials. The error term includes a Gaussian noise term, where $\varepsilon \sim \mathrm{N}(0, \sigma=.01)$, representing that the weight is not directly observable and is corrupted by uncertainty. Each of the four cards $\left(\mathrm{C}_{\mathrm{t}}\right)$ was associated with a weight $\left(\mathrm{W}_{\mathrm{t}}\right)$ representing the card's association with the current outcome. The weights of the present cards were summed for the current weight of the group of cards $\left(\mathrm{G}_{\mathrm{t}}\right)$. Finally, choice behavior was modeled via a Softmax function:

$$
\mathrm{P}\left(\text { choice }_{\mathrm{j}} \mid G_{t}^{1} G_{t}^{2}\right)=\frac{\exp \left(\beta *\left(G_{t}^{j}\right)\right)}{\exp \left(\beta *\left(G_{t}^{1}\right)\right)+\exp \left(\beta *\left(G_{t}^{2}\right)\right)}
$$

The Softmax function describes the probability of the current choice given the weights of the current groups of cards, which is governed by the inverse temperature parameter, $\beta$, in which higher values contribute to greater stochasticity and lower values contribute to more deterministic choice behavior.

Free parameters were first fit to participants' choices and the outcomes experienced by using maximum likelihood estimation (MLE) and subsequently fit using maximum a posteriori (MAP) estimation. MAP estimation regularizes the parameter estimates by using priors on the possible range of parameters. The priors were defined as the mean and standard deviation from the maximum likelihood estimation. Participant BICs were summed across all participants within each condition for each model. BIC estimation appears as:

$B I C=-2 * L L+\mathrm{k}^{*} \ln (\mathrm{n})$

Models' BICs were subtracted by the base model BIC and compared to base model (i.e. $\Delta$ $\mathrm{BIC}=0$ ), to determine how if model representing a hypothesis about a particular learning process exceeded model performance of base model, which is agnostic about any specific learning process.

Table 17. Six competing reinforcement learning models that were tested.

\begin{tabular}{|l|l|l|}
\hline Model type & Description & Mechanism \\
\hline Base & $\begin{array}{l}\text { No hypothesis about } \\
\text { learning }\end{array}$ & NA \\
\hline Asymmetrical & $\begin{array}{l}\text { Tests for whether there } \\
\text { is differential learning } \\
\text { based on the type of } \\
\text { feedback experienced } \\
\text { (e.g. more steal than } \\
\text { expected so learning is } \\
\text { greater) }\end{array}$ & First-order \\
\hline Categorical Learning & $\begin{array}{l}\text { Tests for whether there } \\
\text { is differential learning }\end{array}$ & First-order \\
\hline
\end{tabular}




\begin{tabular}{|l|l|l|}
\hline & $\begin{array}{l}\text { based on the type of } \\
\text { stimulus present (e.g. } \\
\text { black face is present so } \\
\text { learning is diminished). }\end{array}$ & \\
\hline $\begin{array}{l}\text { Asymmetrical } \\
\text { Learning with } \\
\text { Categories (Original } \\
\text { predicted model) }\end{array}$ & $\begin{array}{l}\text { Tests whether there is } \\
\text { differential learning } \\
\text { based on both the } \\
\text { stimulus present and } \\
\text { the type of feedback } \\
\text { (e.g. there is a black } \\
\text { face and it is more steal } \\
\text { than expected so } \\
\text { learning is enhanced). }\end{array}$ & First-order \\
\hline Time-Based Decay & $\begin{array}{l}\text { Learning rate decreases } \\
\text { linearly as a function of } \\
\text { time }\end{array}$ & Second-order \\
\hline $\begin{array}{l}\text { Exponential-Based } \\
\text { Decay }\end{array}$ & $\begin{array}{l}\text { Learning rate decreases } \\
\text { exponentially as a } \\
\text { function of time }\end{array}$ & Second-order \\
\hline
\end{tabular}

\section{Results}

Ten competing models were fit to participant choices (see Supplemental Table 17). These models were theory-driven and were designed to test competing hypotheses regarding whether learning processes are best characterized by learning rates that are influenced by trial-level features (e.g., asymmetrical learning rates for stimuli) or by learning rates that are disrupted more cumulatively over time. To capture the cumulative effect, we included models with a decay parameter. The decay parameter allows feedback from early trials to exert a larger influence over future predictions, whereas later feedback has an increasingly smaller influence (Kruschke \& Johanses, 1999). Decay parameters can influence learning if they are too small or too large. Decays that are too large may impinge on learning as they suppress learning rates over time, resulting in small updates and a general failure to incorporate feedback. Prior research investigating the role of decayed learning rates (i.e. annealing) in probabilistic category learning often frame this influence in terms of people persistently relying on old information despite new and better information being available (i.e. error discounting; Blair \& Homa, 2005). Conversely, decays that are too small may impinge on learning as they fail to suppress learning rates, resulting in too large of updates that overshoot. Thus, optimal learning may be best characterized by a medium sized decay parameter that allows feedback to update learning rates but limits its ability to exert an overly large influence.

Comprehensive tests of these reinforcement learning models indicated that the model that best explained behavior across conditions was an exponential-based decay (ED) model. To further validate this model, we estimated a logistic mixed model by regressing accuracy onto the model generated action probabilities (AP), using leave-one-out cross validation to reduce overfitting. This model revealed that AP significantly predicted accuracy $(b=4.06,95 \% C I=$ $[3.97,4.13], S E=.04, z=99.11, p<.0001)$. Importantly, while the ED model performed better 
than all other models for all other conditions, it was the worst fitting model in the Crime Face condition relative to all other conditions, suggesting that learning within a context that elicits stereotypical associations is not fully captured by the ED parameter. The poor model fit for the Crime condition parallels the plateauing of learning in the predicted estimates of accuracy across trials. That is, learning plateaus earliest for participants in the Crime condition $(M=204, S D=$ 63.5), which is consistent with ED as the worst fitting model, and plateaus latest for participants in the Weather condition $(M=178, S D=58.2)$, which is consistent with ED as the best fitting model for Weather. This finding suggests that learning decrements in the Crime condition may be partly attributable to second-order disruptions during feedback that accumulate over time rather than first-order disruptions at the trial level.

If learning decrements in the Crime condition are due to disruptions in feedback, then this should manifest in decay parameters that are higher than the other conditions, representing the idea that participants are failing to optimally incorporate feedback. Notably, we found that participants in the Crime condition had the highest average decay parameter $(M=.11 ; S D=.09)$, whereas participants in the Weather condition had a moderate average decay $(M=.1 ; S D=.08)$ relative to participants in the Steal Clouds and Weather Faces conditions $(M=.09 ; S D=.08, M=$ $.09 ; S D=.08$, respectively). This suggests that learning decrements in the Crime condition are attributable to the suppression of learning rates that fail to fully integrate feedback throughout the task. Together, these findings provide converging evidence that learning decrements in the Crime Face condition are attributable to second-order disruptions due to taxed cognitive functioning.

Supplemental Figure 4. Model comparison: the statistical fit of the exponential decay learning model as a function of condition. Higher BICs indicate worse performance. The model performed best for participants in the Weather condition and worst for participants in the Crime condition.

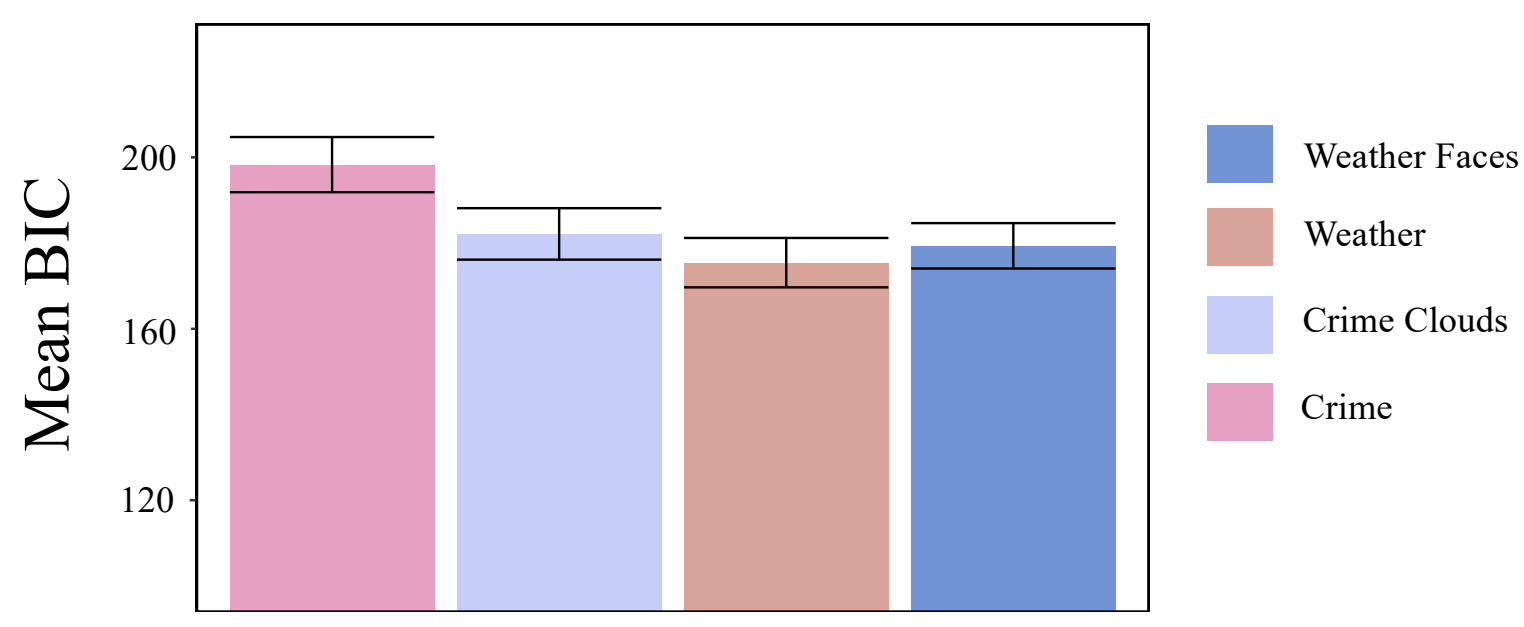

Supplemental Table 18. Model fit indices for competing models in study 2 (Difference from Base) using maximum likelihood estimation. 
STEREOTYPES DISRUPT LEARNING

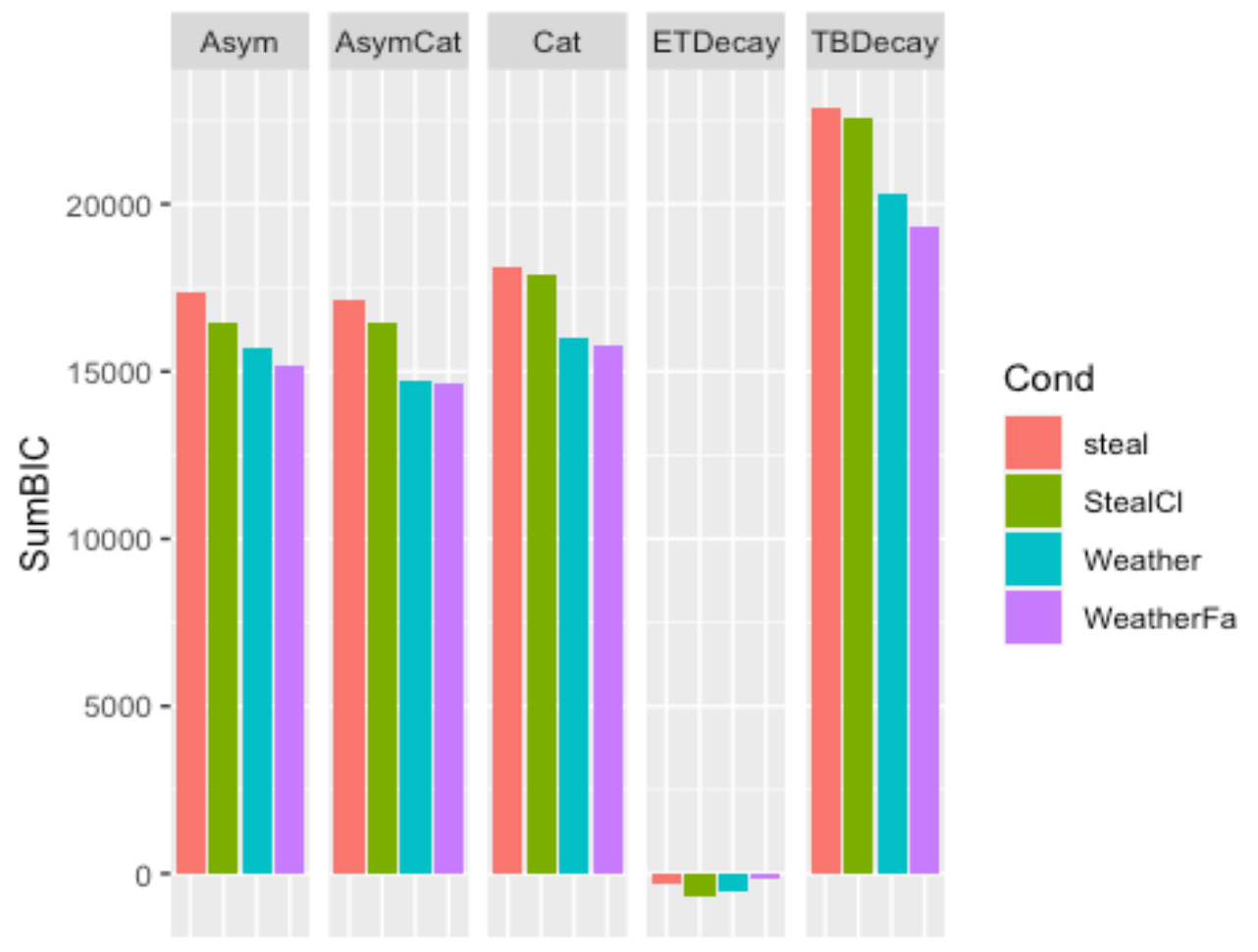

\section{Discussion}

Computational models of participant choices revealed that learning is best characterized by a model where learning rates decay exponentially over time, providing converging evidence that learning disruptions do not occur on a trial-level basis from the mere presence of stereotype congruent or incongruent stimuli. This is consistent with behavioral results which found no difference in accuracy due to trial-level disruptions (e.g., race of stimuli, stereotype congruency reaction time, card pattern predictiveness). Rather, learning seems to be disrupted on a more cumulative and temporally extended basis due to the suppression of learning rates that were largest for participants in the Crime condition. We speculate that these cumulative learning decrements may be due to taxed cognitive functioning as participants attempt to inhibit the expression of stereotype-consistent responses. Specifically, IMS may encourage participants to fail to integrate feedback in environments where they are encumbered by race-related preoccupations.

\section{Reinforcement learning models in Study 3}

Behavioral results provide evidence that learning decrements in the Crime Face condition are attributable to cumulative effects due to concerns over appearing prejudice. However, IMS was not a significant predictor of accuracy for participants in the Athletic condition, suggesting that learning decrements may be attributable to alternative mechanisms. For instance, people may be more likely to apply stereotypes in contexts that they perceive to be more innocuous and are therefore less concerned over expressing prejudice. While we did not find any behavioral 
indication of stereotype application (e.g., effects of the race of the stimuli on accuracy or choices), the RL models provide a more nuanced method to test this alternative mechanism.

In addition to examining the different potential mechanisms underlying learning in the Athletic condition, this analysis provides an opportunity to replicate the finding that learning decrements in the Crime condition is best characterized by an exponential decay model.

\section{Method}

The reinforcement learning models were fit identically to before, such that ten competing models were fit to participant choices (see Table 17). These models were designed to test competing hypotheses regarding whether learning processes in the Crime Face and Athletic Face condition are best characterized by learning rates that are influenced by trial-level features (e.g., asymmetrical learning rates for stimuli and feedback) or by learning rates that are disrupted more cumulatively over time.

\section{Results and Discussion}

Comprehensive tests of these reinforcement learning models indicated that the model that best explained behavior in both the Crime and Athletic condition was an exponential-based decay (ED) model (see Supplemental Table 18). To further validate this model, we estimated a logistic mixed model by regressing accuracy onto the model generated action probabilities (AP), using leave-one-out cross validation to reduce overfitting. This model revealed that AP significantly predicted accuracy $(b=.26,95 \% C I=[3.97,4.13], S E=.012, z=20.4, p<.0001)$. While these findings replicate Study $2 \mathrm{~b}$ and suggest that learning is best characterized by cumulative-level effects, it opens up new questions regarding learning mechanisms in the Athletic condition. For instance, even though we found no behavior evidence that learning decrements were attributable to stereotype application for participants in the Athletic condition, the Athletic condition performed better than the Crime condition across both the trial-level and cumulative-level models, suggesting greater disruptions in the Crime condition. Moreover, even though we found no evidence that IMS was associated with accuracy in the Athletic condition, the best fitting model was an ED model. Together, these findings may reflect similar kinds of learning processes, but different degrees of disruption. In other words, similar mechanisms may underlie the disruptions across negative and positive stereotypes, but negative stereotypes may elicit larger degrees of disruption.

Supplemental Table 19. Model fit indices for competing models for Study 3 (Difference from Base) using maximum likelihood estimation. 
STEREOTYPES DISRUPT LEARNING

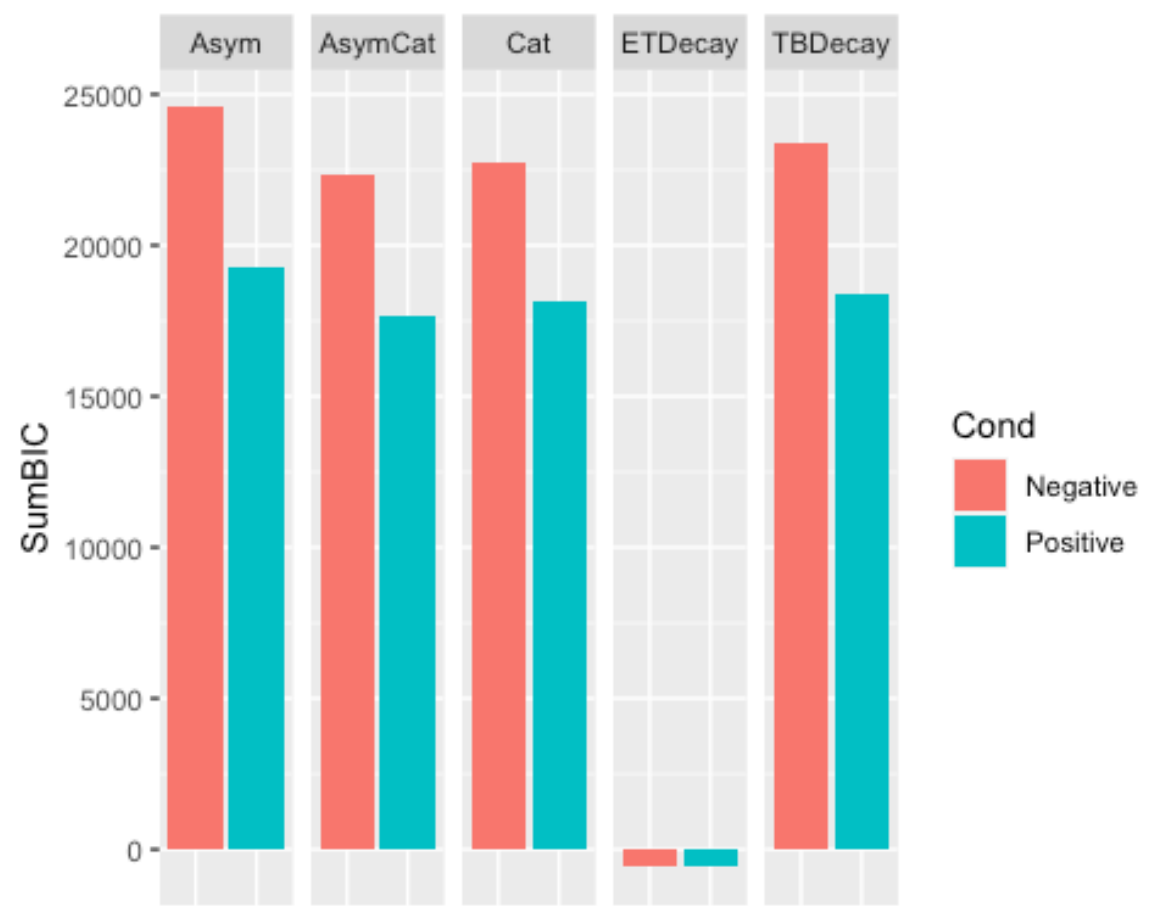

\section{References cited}

Daw, N., O'Doherty, J., Dayan, P. et al. Cortical substrates for exploratory decisions in humans. Nature 441, 876-879 (2006). https://doi.org/10.1038/nature04766

Rescorla, R., \& Wagner, Allan. R. (1972). A theory of Pavlovian conditioning: Variations in the effectiveness of reinforcement and nonreinforcement. In Classical conditioning: Current research and theory.

Kruschke, J. K., \& Johansen, M. K. (1999). A model of probabilistic category learning. Journal of Experimental Psychology: Learning, Memory, and Cognition, 25(5), 1083-1119. https://doi.org/10.1037/0278-7393.25.5.1083

Blair, M., \& Homa, D. L. (2005). Integrating Novel Dimensions to Eliminate Category Exceptions: When More Is Less. Journal of Experimental Psychology: Learning, Memory, and Cognition, 31(2), 258-271. https://doi.org/10.1037/0278-7393.31.2.258 